Elsevier required licence: (C) <2019>. This manuscript version is made available under the CC-BY-NCND 4.0 license http://creativecommons.org/licenses/by-nc-nd/4.0/

The definitive publisher version is available online at

[https://linkinghub.elsevier.com/retrieve/pii/S2352467718303825] 


\title{
A Nodal Approach based State-Space Model of Droop-based Autonomous Networked Microgrids
}

\author{
Mohsen Eskandari ${ }^{\mathrm{a},}$, Li Li $^{\mathrm{a}}$, Mohammad H. Moradi ${ }^{\mathrm{b}}$, Pierluigi Siano ${ }^{\mathrm{c}}$ \\ ${ }^{a}$ Faculty of Engineering and Information Technology, University of Technology Sydney, Australia \\ ${ }^{\mathrm{b}}$ Department of Electrical Engineering, Bu-Ali Sina University, Hamedan, Iran \\ ${ }^{c}$ Department of Management \& Innovation Systems, University of Salerno, Salerno, Italy
}

\begin{abstract}
As the requirement of expensive and unreliable high band-width communication infrastructure is obviated, decentralized droop-like control method has been considered for power sharing implementation in autonomous microgrids (MGs). To this end, the power network is regarded as a communication link and voltage variables (magnitude and frequency) as control signals. This, however, reduces the stability margin of islanded MGs due to the interaction of droop controllers through the power network. Lack of inertia of droop-controlled power converters and low $X / R$ ratio of interconnecting power lines intensify this interaction which may lead to the instability of Networked MGs (NMG). On the other hand, the existing parallel-based small signal model of MGs is inadequate to represent this interaction, as the adopted common-based reference frame (RF) is not applicable in islanded NMGs. This issue is investigated in this work in details and, inspired from power flow equations, a local RF is proposed to improve the small-signal model accuracy. Droop controllers are also correlated through the power flow equations to properly model their interaction through the power network. Moreover, the state-space model is developed in a fully decentralized approach which does not rely on any converter for any specific role. Eigenvalue analysis and simulation in MATLAB $\backslash$ SIMULINK platforms are executed to evaluate the effectiveness and accuracy of the proposed model.
\end{abstract}

Index Terms—Droop Control; Dynamic Stability; Microgrid; Power Sharing; Small Signal model; State Space Model; Voltage Regulation.

\section{INTRODUCTION}

The microgrid (MG) concept has been proposed to gain the full advantages of renewable energy resources (RESs) penetration into power systems [1]-[2]. Among all benefits MGs provide, islanding ability is a remarkable feature, which enhances the reliability of delivered power to sensitive customers. Besides, electrification of countryside and remote areas is becoming reasonable by reducing costs and addressing environmental concerns related to transmission and distribution lines [3]. Autonomous operation of MGs, isolated from the main power grid, needs novel control schemes in relation to power sharing and voltage regulation [4]-[5]. MGs consist, indeed, of distribution generation (DG) units, such as RESs, energy storage systems (EESs) and micro-sources (MSs), which are connected to the grid via power electronic converters [6]-[7]. Furthermore, the small scale of DG units requires the control system adopted in the MG to be accurate to prevent them from overloading and to avoid voltage deviations [8]. Some control methods based on high band-width communication infrastructures have been suggested. Despite of the accuracy and stability of such control systems, the long distance between DG units makes them expensive and not much reliable [9].

Utilizing the power network as communication links and its variables as control signals is a big ambition which is realized by droop-like control methods. Conventional droop control technique is implemented inherently in large synchronous generators in which the rotational speed of the rotor is drooped following an increase in the output active power. This phenomenon is employed to implement power sharing among synchronous generators by setting the corresponding droop gains. This method is adopted in $f-P$ and $V$ - $Q$ droop-like control methods by which the frequency and voltage magnitude of the converter's output voltage are drooped by an increase in the active and reactive power proportionally to the corresponding droop coefficients [10]. No need of communication infrastructure, easy implementation and plug-\&-play capability are the significant characteristics of droop control. However, droop control functioning dependency on the power network and voltage variables, which has made it as a promising control method for MGs, also causes, as drawbacks of the droop control, inferior dynamic performance and stability [11]. Besides, lack of spinning reserve, low rating of DG units, low $\mathrm{X} / \mathrm{R}$ ratio of the power network and converters with fast response (lack of inertia) make the stability margin of droop control much smaller [12]. So small signal modeling of the droop-based control system is essential to evaluate its performance and more importantly the dynamic stability. The droop control is situated in the primary level of the hierarchical control structure with special responsibility on MG stabilization [13]-[14]. Hence, in many of the works done to improve the droop control performance in MGs, small signal models are established to prove that the presented approach is effective [11], [12], [15]-[38]. However, these models are partial and incomplete.

In [39] a pretty complete, yet not comprehensive model is developed in $d-q$ synchronous reference frame (RF), which is used in [40]-[42] as well. The model is straightforward for parallel converters, but not for networked MGs (NMGs). Besides, this model is developed based on common reference frame (CRF), which is not an accurate model due to the absence of the slack bus in islanded NMGs [43]-[46]. This issue is investigated in this work in details. Furthermore, in the case where the converter of CRF is lost due to contingency, the model accuracy also will be lost.

On the other hand, in the dynamic phasor models [47]-[49], the dynamics of voltage source inverters' (VSIs') inner control loops, i.e. voltage and current control loops, are ignored which results in an inaccurate model. It is, indeed, demonstrated in this 
work that the inner voltage and current control loops, which are designed to compensate the LC filter dynamics and to reject disturbances (coming from events in the DC bus or to load changes) must be considered in the model; otherwise the model is not accurate and thus not reliable. This important issue, i.e. the voltage magnitude dynamics, has not been taken into account in the mentioned dynamic phasor-based models. As a result, the cross coupling between $f-P$ and $V$ - $Q$ control loops in weak power networks (with low $\mathrm{X} / \mathrm{R}$ ratio) is not appropriately modelled in those models.

Furthermore, the key factor which must be incorporated into the small signal model is the fundamental principle of droop control, i.e. using voltage variables as control signals which operate through the power network. This is the idea behind the expression of "interaction of droop controllers through the power network" which is emphasized in this work. It is demonstrated that the critical dominant modes in the MGs are related to the droop controllers, which determine the dynamic behavior of the system. So, the interaction of droop controllers must be considered in developing the small signal model.

This paper presents a systematic and comprehensive state space model (including DC link model) for droop-based NMGs. The novel contributions and superiority of the proposed power network-based (NT-based) model in comparison with the conventional parallel-based (PL-based) model [39] are outlined as follows:

- The developed model is applicable to NMGs with arbitrary power network topologies;

- The presented method is developed with fully decentralized operation of converters and without allocating a special responsibility to any converter. This feature enables plugand-play stability analysis as well;

- The inaccuracy of the classical PL-CRF-based model is investigated in detail (Highlight2) followed by proposing local-based RF (inspired from power flow equations) to improve the accuracy of the proposed model. Eigen-analysis is conducted to prove that the adapted (NT-based) model exhibits significant improvements in comparison to the PLbased model and also in discovering unstable regions more accurately;

- The small-signal models of individual converters are correlated through the power flow equations, developed in the $d-q$ RF so that the interaction of droop controllers through the power network is properly modeled (Highlight1). Moreover, state-space models of all the components are modified to be incorporated in the model;

- Based on the modified model presented in this work, it is found that, in addition to droop gains, the $\mathrm{X} / \mathrm{R}$ ratio of feeder impedance in the NMG (cross-coupling effect), power network topology, phase angle difference as consequence of increasing load demand, cut-off frequency of the low pass filters, and interaction of droop controllers are the most important factors influencing the stability margin of NMGs, while electromagnetic transient of power lines is not effective in critical mode oscillations and can be ignored;

- The proposed model is a reduced-order model (Highlight3) that models power network via power flow equations and ignoring power line dynamics. It is also proved that the reduced-order model is almost as accurate as its full-order version.

In Section II, power flow study is introduced briefly to highlight the importance of the modelling methodology. The expression of "interaction of droop controllers" and issues related to the conventional model are presented in Section III. The small signal model is developed in Section IV. The simulation results and eigen-analysis study are presented in Section V, followed by the conclusions drawn in Section VI.

\section{POWER FLOW STUDY}

Power flow study focuses on the power system variables, i. e. voltage magnitude and phase angle of the voltage at power network nodes and active/reactive power flowing through the interconnecting lines. As these variables are used in the droop control rules, it is desirable to know how these variables are correlated to each other in the power network. The power flowing from $i^{\text {th }}$ bus to $j^{\text {th }}$ bus is obtained as:

$$
\begin{aligned}
& P_{i j}=\frac{V_{i}}{Z_{i j}}\left[\left(V_{i}-V_{j} \cos \left(\delta_{i j}\right)\right) \quad V_{j} \sin \left(\delta_{i j}\right)\right]\left[\cos \left(\theta_{i j}\right) \sin \left(\theta_{i j}\right)\right]^{T} \\
& Q_{i j}=\frac{V_{i}}{Z_{i j}}\left[-V_{j} \sin \left(\delta_{i j}\right) \quad\left(V_{i}-V_{j} \cos \left(\delta_{i j}\right)\right)\right]\left[\cos \left(\theta_{i j}\right) \quad \sin \left(\theta_{i j}\right)\right]^{T}
\end{aligned}
$$

where $V_{i} \& \delta_{i}$ are voltage magnitudes and phase angle at bus $i$, respectively; $Z_{i j} \& \theta_{i j}$ are the magnitude and the phase angle of the $i j^{\text {th }}$ interconnecting power line impedance, respectively; $P_{i j}$, $Q_{i j}$, are the active power and the reactive power flowing through $i j^{\text {th }}$ interconnecting line, and $\delta_{i j}=\delta_{i}-\delta_{j}$. By expanding (1)-(2), (3)-(4) can be developed as:

$$
\left[\begin{array}{c}
P_{i j} \\
Q_{i j}
\end{array}\right]=\frac{V_{i}}{Z_{i j}}\left(\left[\begin{array}{cc}
V_{i} & 0 \\
0 & V_{i}
\end{array}\right]-\Psi\left(\delta_{i j}\right)\left[\begin{array}{cc}
V_{j} & 0 \\
0 & V_{j}
\end{array}\right]\right)\left[\begin{array}{c}
\cos \left(\theta_{i j}\right) \\
\sin \left(\theta_{i j}\right)
\end{array}\right]
$$

where $\Psi\left(\delta_{i j}\right)=\left[\begin{array}{cc}\cos \left(\delta_{i j}\right) & -\sin \left(\delta_{i j}\right) \\ \sin \left(\delta_{i j}\right) & \cos \left(\delta_{i j}\right)\end{array}\right]$

The power balance equations at each bus are:

$0=-P_{i}+\sum_{j=1}^{n} \Gamma_{i j} P_{i j}+P_{L D i}+P_{L D C o n, i}$
$0=-Q_{i}+\sum_{j=1}^{n} \Gamma_{i j} Q_{i j}+Q_{L D i}+Q_{L D C o n, i}$

where $P_{i}$ and $Q_{i}$ are injected real and reactive powers to bus $i$, $P_{L D i}$ and $Q_{L D i}$ are related to the $R L$ load at bus $i, P_{L D C o n, i}$ and $Q_{L D C o n, i}$ are the constant $P$ and $Q$ loads at bus $i, \Gamma_{i j}$ is the $i j^{\text {th }}$ entry of the MG layout matrix which is 1 if buses $i$ and $j$ are connected and 0 otherwise, $n$ is the number of buses. From (5)-(6) the output current of bus $i$ is obtained as:

$i_{o d}=\left(\sum_{j=1}^{n} \Gamma_{i j} i_{i j}\right)+i_{L D i}+i_{L D C o n i}$

where $i_{L D i}$ denotes the local RL load current at bus $i$, and $i_{L D C o n i}$ is the constant load current at bus $i$ which is considered as disturbances in this paper. 


\section{Issues Related TO THE CONVENTIONAL PARALLEL- BASED SMALL SIGNAL MODEL OF MGS AND SOLUTIONS}

\section{A. Droop controllers}

The $f-P$ and $V$ - $Q$ droop-like controllers are adopted for power sharing implementation and voltage regulation in isolated MGs. By using them, the frequency and voltage magnitude of VSIs are dropped proportionally to their output active power and reactive power, respectively (8)-(9) [13]. The objectives behind droop control rules are to dispatch the active power among DG units and to prevent circulating reactive current between them [10]:

$$
\begin{aligned}
& \omega_{i}=\omega_{0}-k_{p i} \tilde{P}_{i} \\
& V_{i}=V_{0}-k_{q i} \tilde{Q}_{i}
\end{aligned}
$$

where $V\left(V_{0}\right)$ and $\omega\left(\omega_{0}\right)$ are the operating (nominal) voltage magnitude and angular frequency, respectively. $\tilde{P}$ and $\tilde{Q}$ are the average active and reactive power obtained from passing instantaneous active and reactive powers $(P$ and $Q)$ through the low pass filter, respectively, $k_{p} \& k_{q}$ are the $f-P$ and $V-Q$ droop gains, respectively and index $i$ denotes the $i^{\text {th }}$ bus. It is worth mentioning here that in the autonomous MGs, the reference values of active and reactive power are considered zero to distribute the demanded load among DG units proportionally to assigned droop gains which, in turn, are specified by the MG energy management system according to the available capacity of generation units or optimally to maximize the MG's energy efficiency.

\section{B. Reference frame}

Since the frequency is a global variable in the $\mathrm{MG}$, active power sharing is implemented accurately so that the power sharing goal, i.e. $k_{p i} \tilde{P}_{i}=k_{p j} \tilde{P}_{j} \forall i, j \in \mathrm{n}$, is established. So, from (8) the frequency is the same at all MG's nodes in steady states. Nevertheless, the frequency dynamics at each node depends on the VSI's output active power dynamics, which in turn, is affected by the power network topology of the MG. As a result, frequency at each node changes with unique dynamics in the MG. Now let refer to the phase angle dynamics. From (8) and noting that $\delta=\int \Delta \omega d t$, the phase angle dynamics at bus $i$ is derived as:

$\delta_{i}=\int\left(\omega_{i}-\omega_{0}\right) d t=\int-k_{p i} \tilde{P}_{i} d t$

Although the steady state value of $k_{p i} \tilde{P}_{i}$ is the same at all MG's nodes, different dynamics of $\tilde{P}_{i}$ for $i=1: n$, leads to different phase angles at individual nodes. This phenomenon is illustrated in Fig. 1. The area under each curve, representing the integral term of $k_{p i} \tilde{P}_{i}$ at bus $i$, specifies the phase angle at the corresponding node. The difference between areas leads to a phase angle difference which is high in the dynamic state and should be considered in the small signal model. As the system goes to steady state the common area becomes higher which makes the phase angles at the MG nodes close to each other. It is worth noting that the phase angle dynamics is important because the sensitivity of the active/reactive power variation with respect to the phase angle is proportional to the square of the voltage magnitude; see (1)-(2).

In the control system of VSI, 3-phase ac variables are transformed to $\boldsymbol{d c}$ variables in the $d-q$ synchronous RF. The $\boldsymbol{d c}$ variables are manipulated in the VSI's control loops and retransformed to the $\boldsymbol{a b c} \mathrm{RF}$ as control signals. On the other hand, as it is investigated earlier, the $f-P$ droop controller performance leads to different RFs at individual generation nodes in the MG. As a result, control variables at individual VSIs are manipulated at different RFs. In developing the small signal model of an MG, it is necessary to link control variables at individual VSIs at a same RF. To this end, the CRF is adopted in the conventional small signal model of MGs presented in [39], which develops a PL-based small signal model of MGs including parallel VSIs. In the PL-based model, the RF of one VSI is considered as CRF. The MG's power network, common bus and common loads are modeled in the CRF and the small signal models of individual VSIs are transformed to the CRF; however, noting that the phase angle of the CRF varies (maybe rapidly) in the islanded mode, these transformations haven't considered the dynamics of the CRF in the literature. As it is represented later in this work, the CRF is not accurate and is not applicable in autonomous NMGs.

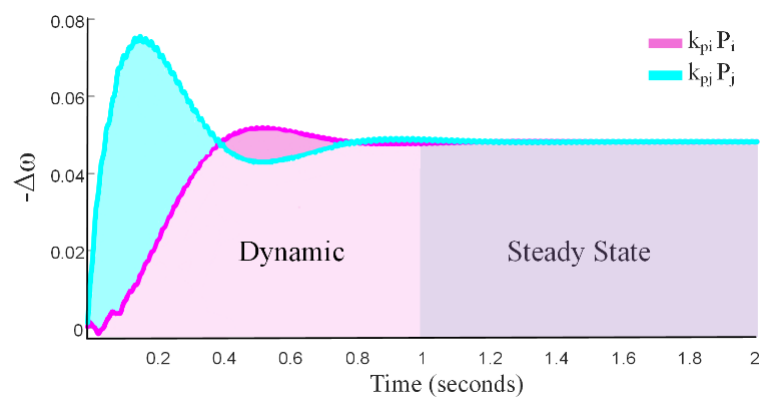

(a)

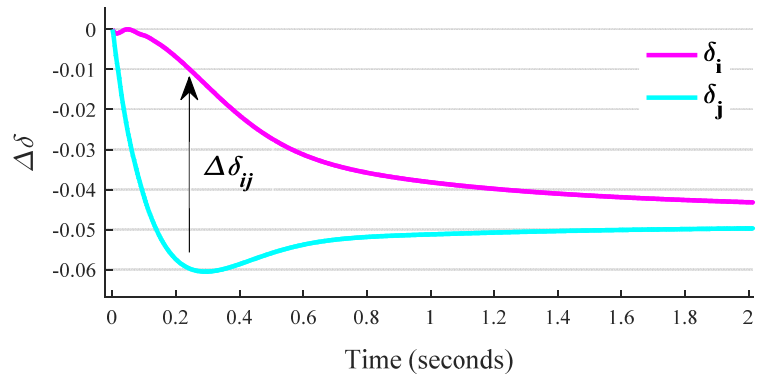

(b)

Fig. 1. (a) Frequency dynamics. (b) Phase angle dynamics.

\section{C.Power network}

Three factors are effective on the current dynamics (and consequently power dynamics) flowing through an interconnecting power line: voltage magnitude, phase angle and power line impedance. Let investigate the dynamic behavior of these items in the $i j^{\text {th }}$ power line:

Phase angle dynamics is specified by $f-P$ droop controller as represented in (10). Further elaboration on (10) together with a low-pass filter (LPF) for the active power results in:

$$
\left\{\begin{array}{c}
\dot{\delta}_{i}=-k_{p i} \tilde{P}_{i}=-k_{p i} \sum_{j=1}^{n} \Gamma_{i j} \tilde{P}_{i j} \\
\tilde{P}_{i j}(\mathrm{~s})=\frac{\omega_{c}}{s+\omega_{c}} P_{i j}(\mathrm{~s})
\end{array}\right.
$$

where the local loads are ignored. Taking only the $i j^{\text {th }}$ power network branch into account, for the sake of clarity, and further 
manipulation on (11-a) results in:

$$
\begin{gathered}
\ddot{\delta}_{i}+\omega_{c} \dot{\delta}_{i}=-k_{p i} \omega_{c}\left(\frac{V_{i}}{Z_{i j}}\left(V_{j} \sin \left(\theta_{i j}\right) \delta_{i j}+\left(V_{i}-V_{j}\right) \cos \left(\theta_{i j}\right)\right)\right) \\
\Rightarrow \ddot{\delta}_{i}+\omega_{c} \dot{\delta}_{i}+k_{p i} \omega_{c}\left(\frac{V_{i} V_{j} \sin \left(\theta_{i j}\right)}{Z_{i j}}\right) \delta_{i}= \\
-k_{p i} \omega_{c}\left(\frac{V_{i}}{Z_{i j}}\left(-V_{j} \sin \left(\theta_{i j}\right) \delta_{j}+\left(V_{i}-V_{j}\right) \cos \left(\theta_{i j}\right)\right)\right)
\end{gathered}
$$

(11-b)

where $\omega_{c}$ is the cutting frequency of LPF, $P_{i j}$ is replaced from the power flow equation (1) assuming $\sin \left(\delta_{i j}\right) \approx \delta_{i j}$ and $\cos \left(\delta_{i j}\right) \approx 1$. Consequently, the time constant of the phase angle dynamics $\left(T_{\delta}\right)$ is obtained as $T_{\delta}=2 / \omega_{c}$.

Power line dynamics is specified by the electromagnetic transient with the time constant of an RL circuit which is $T_{L}$ $=L / R$. In microgrids, the $\mathrm{X} / \mathrm{R}$ ratio of the feeder is low, approximately one $\left(\omega_{0} L / R=1\right)$, which means $T_{L}=L / R=1 / \omega_{0} \approx 2 e$ 3 .

Voltage magnitude dynamics is determined by the $V-Q$ droop controller at the corresponding node. With a LPF for the reactive power, the $V$ - $Q$ droop loop dynamics is given as:

$$
\left\{\begin{array}{c}
V_{i}-V_{0}=-k_{q i} \tilde{Q}_{i}=-k_{q i} \sum_{j=1}^{n} \tilde{Q}_{i j} \\
\tilde{Q}_{i j}(\mathrm{~s})=\frac{\omega_{c}}{s+\omega_{c}} Q_{i j}(\mathrm{~s})
\end{array}\right.
$$

where the local loads are ignored. Taking only the $i j^{\text {th }}$ power network branch into account and further manipulation on (12a) results in:

$$
\begin{aligned}
& \dot{V}_{i}+\omega_{c} V_{i}-\omega_{c} V_{0}=-k_{q i} \omega_{c}\left(\frac{V_{i}}{Z_{i j}}\left(\left(V_{i}-V_{j}\right) \sin \left(\theta_{i j}\right)-V_{j} \cos \left(\theta_{i j}\right) \delta_{i j}\right)\right) \\
& \Rightarrow \dot{V}_{i}+\omega_{c}\left(1+\frac{k_{q i}}{Z_{i j}}\left(\left(V_{i}-V_{j}\right) \sin \left(\theta_{i j}\right)-V_{j} \cos \left(\theta_{i j}\right) \delta_{i j}\right)\right) V_{i}=\omega_{c} V_{0}
\end{aligned}
$$

where $Q_{i j}$ is replaced from the reactive power flow equation (2). Consequently, the time constant of the voltage magnitude dynamics $\left(T_{v}\right)$ is obtained as $T_{v} \approx 1 / \omega_{c}$.

The following facts can be conducted from (11)-(12):

1. The cut-off frequency of low pass filter significantly influences the dynamic performance of the droop-based control system in MGs.

2. The time constant of the interconnecting power line electromagnetic transient is 50 times faster than the $f-P$ droop loop and 25 times faster than the $V$ - $Q$ droop loop dynamics (for $\omega_{c}=10$ ). So the critical dominant modes of the power flow dynamics through the power network are determined by the droop control loop located at power network nodes and the transient of interconnecting power line can be ignored. It is quite normal as the droopcontrolled VSIs are intended to mimic conventional synchronous generators' behavior, determining the dominant oscillations of the bulk power systems.

3. It is proposed that, in NMGs with low $\mathrm{X} / \mathrm{R}$ ratio, low pass filters with lower cut-off frequency $\left(\omega_{c}=15 \mathrm{rad} / \mathrm{s}\right)$ are adopted to decouple the dynamics of droop controllers from the feeder dynamics, which improves the stability margin of the MG control system. This is investigated through the eigen-analysis and simulation in Section V.

4. In addition to the $V$ - $Q$ droop loop, VSI's inner loops are also effective in the voltage magnitude dynamics. The VSI's inner current and voltage control loops are designed to modify the LC filter dynamics in disturbance rejection and also to compensate the voltage fluctuations at the DC link (caused by changes in the produced or demanded power). To this end, equation (12-b) should be modified as:

$$
\dot{\bar{V}}_{i}+\omega_{c} \bar{V}_{i}+k_{q i} \omega_{c}\left(\frac{V_{i}}{Z_{i j}}\left(\left(V_{i}-V_{j}\right) \sin \left(\theta_{i j}\right)-V_{j} \cos \left(\theta_{i j}\right) \delta_{i j}\right)\right)=\omega_{c} V_{0}(12-\mathrm{c})
$$

Where in s-domain $\bar{V}_{l}(s)=\Theta^{-1}(s) V_{i}(s), \bar{V}_{l}$ is the voltage magnitude determined by the $V-Q$ droop controller at bus $i$, and $\Theta(s)$ represents the dynamic model of VSI's inner loops. Although, dynamics of the VSI's inner loops have been considered in the developed small signal model (in $d$ $q \mathrm{RF}$ ) in this paper, it is beyond the scope of this work to develop a dynamic phasor model by taking the VSI's dynamics into account in (12-c), which is the major shortcoming of dynamic phasor models in [47]-[49]. The closed loop dynamics of the VSI's inner loops along with the LC filter determines complex-conjugate-pair critical dominant poles as illustrated in the eigen-analysis in the simulation section.

5. The dynamics of voltage magnitude and phase angle at one node of an interconnecting power line influence the dynamics of those at the other nodes.

The $5^{\text {th }}$ fact above leads to the following highlight.

Highlight1: Fig. 2 shows a schematic diagram of the NMG's network graph in which the droop-based converters are located at the graph vertices. The graph's weighted edges denote the interconnecting power lines with a given impedance. Without loss of generality, considering the $i j^{\text {th }}$ edge and according to the droop control model, magnitude and phase angle of the voltage at the power line nodes are dynamically controlled with the fluctuation of flowing active and reactive power through the power line, $P_{i j}$ and $Q_{i j}\left(-P_{i j}\right.$ and $\left.-Q_{i j}\right)$ at $i^{t h}\left(j^{t h}\right)$ node. On the other hand, the power fluctuation through the network edges is a function of the voltages magnitudes and phase angles at network nodes (see (3)-(4)). This feature reveals the interaction of controllers at the nodes of the interconnecting power line via the flowing power. This idea is applicable to all the droop controllers which interact to each other in the NMG through the power network and should be modeled properly in the small signal model. To this end, the power flowing through the network should be inserted into the small signal model as function of nodal information, which are droop control signals (voltage magnitude and phase angle) as presented in (13).

$(P, Q)_{i j}=f_{i j}\left(V_{i} \angle \delta_{i}, V_{j} \angle \delta_{j}, Z_{i j} \angle \theta_{i j}\right)$, for $j=1: n$

In this way, since voltage magnitude and phase angle are considered as state variables (in the proposed model), controller behavior at the adjacent buses of the $i^{\text {th }}$ controller directly appears in the $i^{\text {th }}$ state model. 


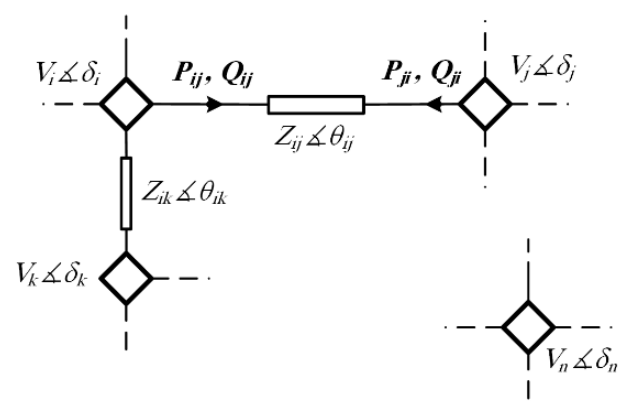

Fig. 2. Interaction of droop controllers through the power network.

Current ( $\left.i_{i j}\right)$ : in order to derive the current flowing from bus $i$ to bus $j$, by applying park transformation to the RL circuit of the $i j^{\text {th }}$ interconnecting power line and ignoring its transient, the relation between current and voltage is obtained as:

$\left[\begin{array}{l}i_{i j d} \\ i_{i j q}\end{array}\right]=\frac{1}{Z_{i j}}\left[\begin{array}{cc}\cos \left(\theta_{i j}\right) & \sin \left(\theta_{i j}\right) \\ -\sin \left(\theta_{i j}\right) & \cos \left(\theta_{i j}\right)\end{array}\right]\left[\begin{array}{l}v_{o d i}-v_{o d j} \\ v_{o q i}-v_{o q j}\end{array}\right]$

where $v_{o, d q i}$ and $v_{o, d q j}$ are $d$-q component of voltage at bus $i$ and $j$, respectively, which are represented at different reference frames and must be transformed to the same RF.

Highlight2: in the conventional PL-based model of MGs, presented in [39], the CRF is taken to correlate $d-q$ variables at different RFs. There are two main drawbacks with this strategy: 1) since there is not any droop controller to specify the phase angle dynamics at the MG's main bus, the PL-based model does not reveal interaction of droop controllers, 2) the idea of CRF comes from the idea of the slack bus in the bulk power systems. The corresponding synchronous generator at the slack bus (normally the largest one) does not participate in the load sharing and its duty is to provide spinning reserve (to hold the balance between production and consumption) and frequency regulation. So the frequency is fixed to a constant known value and the phase angle dynamics at the slack bus is negligible. In MGs, however, this is not profitable and not applicable to assign one DG unit as reserve due to the small scale of DG units [43]-[46]. So, representing the MG's main bus, power network and common load models in an RF of a given converter (with nonzero droop gain), as implemented in [39]-[42], reduces the accuracy of the model, as the effects of other droop controllers on the phase angle dynamics in the MG's common bus is ignored. This issue is illustrated in Fig. 3. In Fig. 3(a), RF of VSI $i$ is taken as the CRF. Fig. 3(b) shows the small signal equivalent of the RF in the MG (please notice that the phase angle variation from the equilibrium point is represented $(\Delta \delta)$, while the phase angle value, and the phase angle dynamics at MG's main bus $\left(\Delta \delta_{B}\right)$, which is negligible, is ignored for the sake of clarity). Since the MG bus is represented in the CRF $\left(D Q_{B}: d q_{i}\right), \Delta \delta_{i j}$ is adopted, instead of $\Delta \delta_{j B}$, to correlate the small signal model of VSI $j$ to the MG's common bus which is not accurate. On the other hand, Fig. 3(c) shows the idea of the slack bus. Putting a large DG unit at bus i with zero or very small droop gain, limits the phase angle variation at bus $i\left(\Delta \delta_{i}\right)$ which makes $\Delta \delta_{i j} \approx \delta_{j B}$. However, the model developed based on CRF is not a precise model and, as mentioned earlier, it is not applicable in MGs due to small scale of DG units and current limits of the semiconductors.

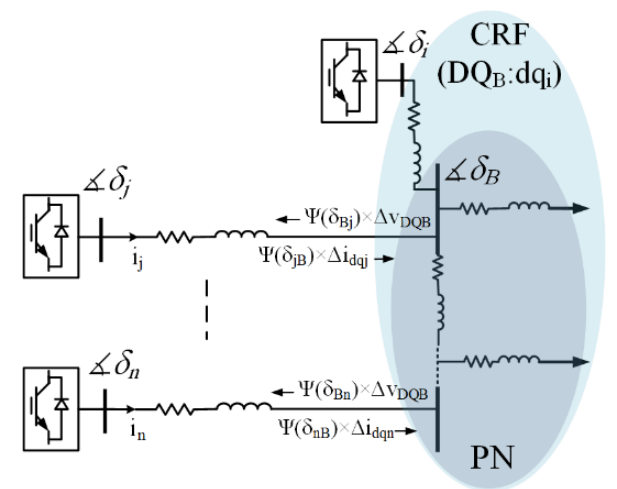

(a)

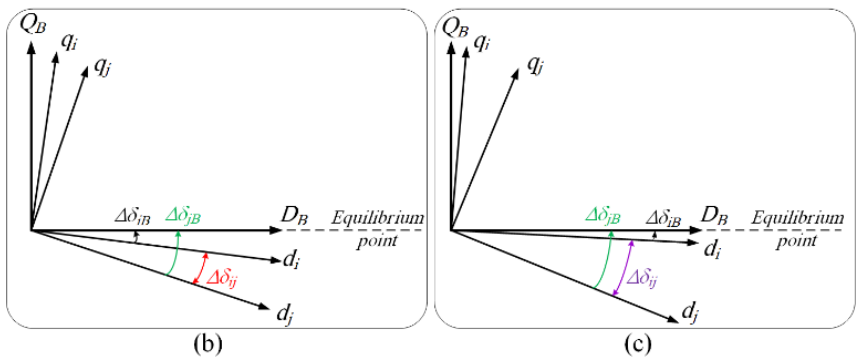

(b)

Fig. 3. Inaccuracy of CRF in the PL-based model. (a) Schematic diagram of CRF applied to PL-based model (RF of VSI $i$ is taken as CRF). (b) Small signal representation of reference frame in the MG. (c) Small signal representation of reference frame including slack bus (bus $i$ ), (Phase angle variation from equilibrium point $(\Delta \delta)$ is represented and $\Delta \delta_{B}$ is ignored).

To address this issue, in this work, the corresponding RF of each DG unit, of which the related state equations are developed, is considered as base and $d-q$ components of voltage at other connected buses are transformed to this frame using the transformation matrix $\Psi(\delta)$, inspired from power flow equations in (3)-(4). So the current flowing from bus $i$ to bus $j$ (14) is modified as following (noting that the operator $\Psi($. satisfies $\left.\Psi\left(\delta_{1}\right) \times \Psi\left(\delta_{2}\right)=\Psi\left(\delta_{1}+\delta_{2}\right)\right)$ :

$\left[\begin{array}{c}i_{i j d} \\ i_{i j q}\end{array}\right]=\frac{1}{Z_{i j}}\left(\Psi\left(-\theta_{i j}\right)\left[\begin{array}{c}v_{o d i} \\ v_{o q i}\end{array}\right]-\Psi\left(\delta_{j i}-\theta_{i j}\right)\left[\begin{array}{l}v_{o d j} \\ v_{o q j}\end{array}\right]\right)$

Equation (15) presents the $d-q$ components of flowing current from the $i^{\text {th }}$ to $j^{\text {th }}$ bus in terms of voltage magnitude and phase angle.

Active and reactive power: to develop $P_{i j}$ and $Q_{i j}$ as function of voltage variables, substituting (15) into (16), the power flowing from bus $i$ to bus $j$ is obtained as (17).

$$
\begin{aligned}
& {\left[\begin{array}{l}
P_{i j} \\
Q_{i j}
\end{array}\right]=\left[\begin{array}{cc}
v_{o d i} & v_{o q i} \\
v_{o q i} & -v_{o d i}
\end{array}\right]\left[\begin{array}{l}
i_{i j d} \\
i_{i j q}
\end{array}\right]} \\
& {\left[\begin{array}{l}
P_{i j} \\
Q_{i j}
\end{array}\right]=\frac{1}{Z_{i j}}\left[\begin{array}{cc}
V_{o d i} & V_{o q i} \\
V_{o q i} & -V_{o d i}
\end{array}\right]\left(\Psi\left(-\theta_{i j}\right)\left[\begin{array}{l}
V_{o d i} \\
V_{o q i}
\end{array}\right]-\Psi\left(\delta_{j i}-\theta_{i j}\right)\left[\begin{array}{l}
V_{o d j} \\
V_{o q i}
\end{array}\right]\right)}
\end{aligned}
$$

\section{State Space Model}

In order to obtain the small-signal model of the whole NMG shown in Fig. 4, firstly the state-space model of the conventional $f-P$ and $V-Q$ droop-based control systems for a DG unit are developed followed by presenting dynamic model of the load bus. Then the power network is modeled by modified 
power flow equations in the $d-q$ frame which are linearized around the equilibrium point. The power flow equations are developed using the state variables of generator and load buses and without defining any new state variable related to the power network, which results in a reduced-order model. Although the MG is considered in a balanced load condition, to eliminates zero components and avoid complexity in presenting the idea of the method, the model is applicable to unbalanced conditions as well. Nevertheless, this does not affect the accuracy of the model. It is supposed that there are $k$ DG units, indexed by $1 \ldots k$, and $n-k$ load buses, indexed by $k+1, \ldots, n$, in the NMG.

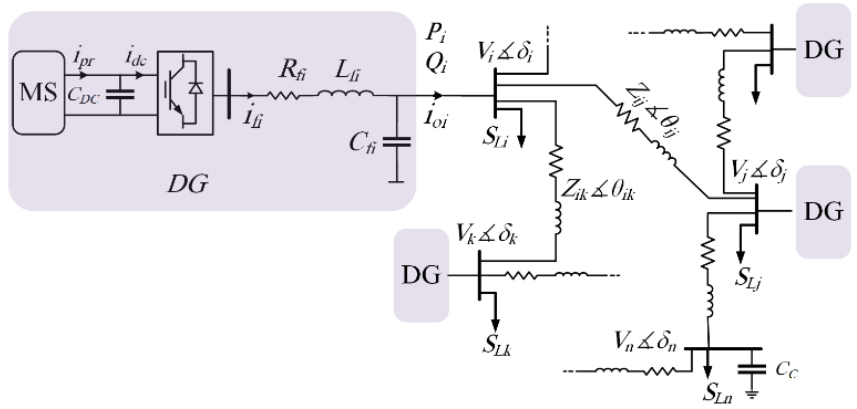

Fig. 4. NMG topology.

\section{A. Generation buses}

The state variables for an individual DG unit are defined as (index $i$ denotes $i^{\text {th }}$ DG unit):

$x_{D G i}=\left[\begin{array}{lllll}x_{D C i}^{T} & x_{D r p i}^{T} & x_{V S l i}^{T} & x_{L C f i}^{T} & x_{L D i}^{T}\end{array}\right]^{T}, i=1, \ldots, k$

where $D C$, Drp, VSI, $L C f$, $L D$ indices denote DC link, droop controller, VSI inner control loops (including virtual impedance), LC filter, and local load.

1) DC link: it is a parallel capacitor connected between MS's output DC voltage and the inverter. DC link capacitor smooths the output DC waveform and helps filtering transients coming from the inverter side. The output power of the inverter follows the load variation quickly because of the lack of inertia and low output impedance of VSIs. On the other hand, MSs have slow response to load tracking which causes DC link voltage drop in severe load variations in autonomous operations of MGs. To model the DC link dynamics, the small signal model of the DC capacitor voltage is obtained as:

$$
\begin{aligned}
& \dot{v}_{D C i}=\frac{1}{C_{D C i}}\left(i_{p r i}-i_{d c i}\right) \\
& i_{d c i}=\sqrt{\left(i_{f d i}{ }^{2}+i_{f q i}{ }^{2}\right)}
\end{aligned}
$$

where $v_{D C i}$ and $C_{D C i}$ are the DC link voltage and capacitor respectively, $i_{p r i}$ is the output current of MS and $i_{d c i}$ is the input DC current to inverter. $i_{d c i}$ is obtained as (20), where $i_{f d, q i}$ are the $d-q$ component of the converter's output current. The converter switching losses are ignored. The MS output current is modeled considering MS's response in load tracking as:

$$
I_{p r i}(s)=\frac{1}{T_{M S i} s+1} I_{d c i}(s)
$$

where $T_{M S i}$ is the $i^{\text {th }}$ MS time constant in load tracking, $I_{p r i}(s)$ and $I_{d c i}(s)$ are the Laplace transformation of $i_{p r i}(t) \& i_{d c i}(t)$, respectively. The DC link state space model, from (19) and (21) and after linearizing (20) at the nominal operating point, is obtained as:

$\dot{x}_{D C i}=A_{D C i} x_{D C i}+A_{D C i, L C i} x_{L C f i}$

where

$$
x_{D C i}=\left[\begin{array}{ll}
\Delta v_{D C i} & \Delta i_{p r i}
\end{array}\right]^{T}
$$$$
x_{L C f i}=\left[\begin{array}{llll}
\Delta i_{f d i} & \Delta i_{f q i} & \Delta v_{o d i} & \Delta v_{o q i}
\end{array}\right]^{T}
$$

$A_{D C i}=\left[\begin{array}{cc}0 & \frac{1}{C_{D C i}} \\ 0 & \frac{-1}{T_{M S i}}\end{array}\right] \quad A_{D C, L C f}=\left[\begin{array}{cccc}\frac{-1}{C_{C i}} \frac{\partial i_{d c i}}{\partial i_{f d i}} & \frac{-1}{C_{C i}} \frac{\partial i_{d c i}}{\partial i_{f q i}} & 0 & 0 \\ \frac{1}{T_{M S i}} \frac{\partial i_{d c}}{\partial i_{f d i}} & \frac{1}{T_{M S i}} \frac{\partial i_{d c}}{\partial i_{f q i}} & 0 & 0\end{array}\right]$

$v_{o d i} \& v_{o q i}$ are the $d-q$ components of measured output voltage (LC capacitor voltage).

\section{2) Droop controller:}

The integral term of angular frequency variation determines the phase angle (10). So the phase angle dynamics is obtained as:

$\dot{\delta}_{i}=-k_{p i} \tilde{P}_{i}$

In order to model the low pass filter transfer function, (with $\omega_{c} /\left(s+\omega_{c}\right)$ from (5)-(6) $\tilde{\mathrm{P}}$ and $\tilde{\mathrm{Q}}$ are obtained as:

$$
\begin{aligned}
& \tilde{P}_{i}(s)=\frac{\omega_{c}}{s+\omega_{c}}\left[\sum_{j=1}^{n}\left(\Gamma_{i j} P_{i j}(s)\right)+P_{L D i}(s)+P_{L D C o n, i}(s)\right] \\
& \tilde{Q}_{i}(s)=\frac{\omega_{c}}{s+\omega_{c}}\left[\sum_{j=1}^{n}\left(\Gamma_{i j} Q_{i j}(s)\right)+Q_{L D i}(s)+Q_{L D C o n, i}(s)\right]
\end{aligned}
$$

where $i$ and $j$ indices denote $i^{t h}$ and $j^{\text {th }}$ buses, respectively, $w_{c}$ is the cutting frequency of the low pass filter. The state space model of the droop controller is represented as:

$$
\begin{aligned}
& \dot{x}_{D r p i}= A_{D r p i} x_{D r p i}+B_{D r p i, L D i} u_{D r p i, L D i} \\
&+B_{D r p i, L D C o n i} u_{D r p i, L D C o n i}+B_{D r p i, P N} u_{D r p i, P N} \\
& \text { where } \quad x_{D r p i}=\left[\begin{array}{lll}
\Delta \delta_{i} & \Delta \tilde{P}_{i} & \Delta \tilde{Q}_{i}
\end{array}\right]^{T} \\
& A_{D r p i}=\left[\begin{array}{ccc}
0 & -k_{p i} & 0 \\
0 & -\omega_{c} & 0 \\
0 & 0 & -\omega_{c}
\end{array}\right] \\
& B_{D r p i, P N}=B_{D r p i, L D i}=B_{D r p i, L D C o n i}=\left[\begin{array}{ccc}
0 & \omega_{c} & 0 \\
0 & 0 & \omega_{c}
\end{array}\right]^{T} \\
& u_{D r p i, L D i}=\left[\begin{array}{ll}
\Delta P_{L D i} \Delta Q_{L D i}
\end{array}\right]^{T} u_{D r p i, L D C o n i}=\left[\begin{array}{ll}
\Delta P_{L D C o n, i} \Delta Q_{L D C o n, i}
\end{array}\right]^{T} \\
& u_{D r p i, P N}=\left[\begin{array}{ll}
\sum_{j=1}^{n} \Gamma_{i j} \Delta P_{i j} & \sum_{j=1}^{n} \Gamma_{i j} \Delta Q_{i j}
\end{array}\right]^{T}
\end{aligned}
$$

The index $P N$ denotes the power network by which the controller interacts with other controllers. So inputs with this index " $P N$ " are modeled based on (15) or (17). The dynamic models of (15)-(17) are presented in Section IVC..

3) VSI control loops: it is composed of two nested control loops:

Voltage control loop: it consists of two control loops: a) droop and virtual impedance loop and b) PI control loop. Virtual impedance loop is added to boost $X / R$ ratio of grid impedance artificially. Please see Fig. 5 for details. The state variables of voltage control loop are defined as: 


$$
\dot{\phi}_{d i}=v_{o d, r e f i}-v_{o d i} \quad \& \quad \dot{\phi}_{q i}=v_{o q, r e f i}-v_{o q i}
$$

where $v_{o d, r e f i}$ and $v_{o q, r e f i}$ are defined by the droop control and virtual impedance loops as in (28)-(29), and $v_{o d} \& v_{o q}$ are the dq components of measured output voltage.

$$
\begin{aligned}
& v_{o d, r e f i}=V_{0}-k_{q i} \tilde{Q}_{i}-R_{v i} i_{o d i}+\omega_{0} L_{v i} i_{o q i} \\
& v_{o q, r e f i}=-R_{v i} i_{o q i}-\omega_{0} L_{v i} i_{o d i}
\end{aligned}
$$

where $R_{v i}$ and $L_{v i}$ are the virtual resistance and inductance, respectively, and $i_{o d i}$ and $i_{o q i}$ are the $d-q$ components of DG unit output current which are obtained from (7).

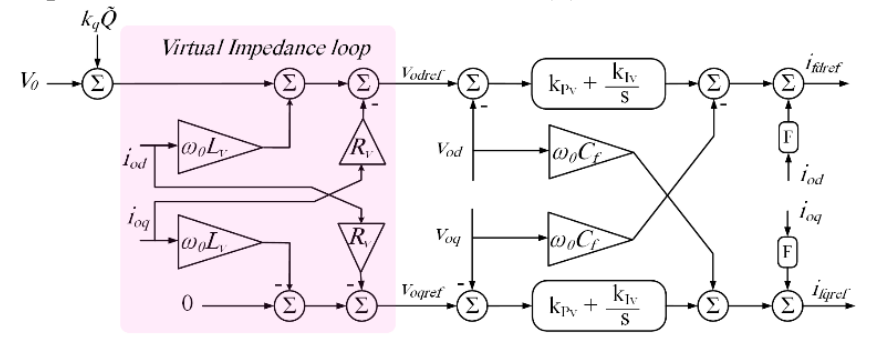

(a)

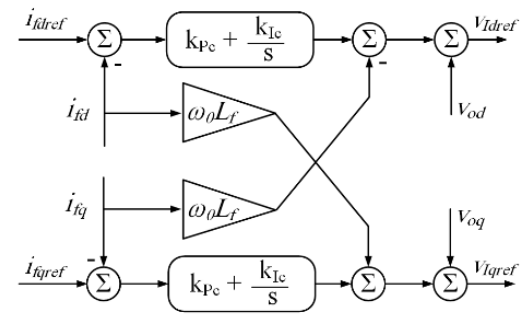

(b)

Fig. 5. Nested control loops of VSI. (a) Voltage control loop. (b) Current control loop.

Current control loop: voltage control loop determines VSI's output current flowing through the filter inductance. Current control loop removes the error between input reference current and measured current by conventional PI controllers. The output of current control loop is the input reference voltage to the pulse-width modulation unit (PWM) to fire the converters' gates. Please notice both the output voltage measured at its output bus and input reference voltage to the converters are at same $d-q$ reference frame determined by $P-f$ droop controller. The state variables of current control loop are defined as:

$$
\dot{\gamma}_{d i}=i_{f d_{r e f i}}-i_{f d i} \quad \& \quad \dot{\gamma}_{q i}=i_{f q_{r e f i}}-i_{f q i}
$$

where $i_{f d, r e f i} \& i_{f q, r e f i}$ are defined by the voltage control loop and $i_{f d i} \& i_{f q i}$ are the measured signals. The state space model representing the VSI control loops is:

$$
\begin{aligned}
\dot{x}_{V S I i}= & A_{V S I i} x_{V S I i}+A_{V S I i, D r p i} x_{D r p i}+A_{V S I i, L C f i} x_{L C f i} \\
& +A_{V S I i, L D i} x_{L D i}+B_{V S I i, P N} u_{V S I i, P N}+B_{V S S i} u_{V S I i}
\end{aligned}
$$

where

$$
\begin{aligned}
& x_{V S I i}=\left[\begin{array}{llll}
\Delta \phi_{d i} & \Delta \phi_{q i} & \Delta \gamma_{d i} & \Delta \gamma_{q i}
\end{array}\right]^{T} \\
& x_{L D i}=\left[\begin{array}{ll}
\Delta i_{L D d i} & \Delta i_{L D q i}
\end{array}\right]^{T}
\end{aligned}
$$

$$
\begin{aligned}
& A_{V S I i}=\left[\begin{array}{cccc}
0 & 0 & 0 & 0 \\
0 & 0 & 0 & 0 \\
k_{I v i} & 0 & 0 & 0 \\
0 & k_{I v i} & 0 & 0
\end{array}\right] \quad A_{V S I i, D r p i}=\left[\begin{array}{ccc}
0 & 0 & -k_{q i} \\
0 & 0 & 0 \\
0 & 0 & -k_{p v i} \cdot k_{q i} \\
0 & 0 & 0
\end{array}\right] \\
& A_{V S S i, L C f i}=\left[\begin{array}{cccc}
0 & 0 & -1 & 0 \\
0 & 0 & 0 & -1 \\
-1 & 0 & -k_{p v i} & -\omega_{0} \cdot C_{f i} \\
0 & -1 & \omega_{0} \cdot C_{f i} & -k_{p v i}
\end{array}\right] \\
& A_{V S I i, L D i}=B_{V S I i, P N}=B_{V S I i}=\left[\begin{array}{cccc}
-R_{v i} & -\omega_{0} L_{v i} & F_{i}-k_{p v i} R_{v i} & -k_{p v i} w_{0} L_{v i} \\
w_{0} L_{v i} & -R_{v i} & k_{p v i} \omega_{0} L_{v i} & F_{i}-k_{p v i} R_{v i}
\end{array}\right]^{T} \\
& u_{V S l, P N}=\left[\sum_{j=1}^{n} \Gamma_{i j} \Delta i_{i j d} \sum_{j=1}^{n} \Gamma_{i j} \Delta i_{i j q}\right]^{T} \quad u_{V S l i}=\left[\begin{array}{ll}
\Delta i_{L D C o n, i d} & \Delta i_{L D C o n, i q}
\end{array}\right]^{T}
\end{aligned}
$$

where $i_{L D d, q}$ are $d-q$ components of $R L$ local loads.

4) $\boldsymbol{L C}$ filter: it is added to the inverter output to remove voltage harmonic components induced because of switching process in the inverter. The equations representing dynamic behavior of the $L C$ filter are obtained in $d-q$ reference frame as:

$$
\begin{aligned}
& \dot{i_{f d i}}=-\frac{r_{f i}}{L_{f i}} i_{f d i}+\omega_{i} i_{f q i}+\frac{1}{L_{f i}} v_{\text {Idrefi }}-\frac{1}{L_{f i}} v_{\text {odi }} \\
& \dot{i_{f d i}}=-\frac{r_{f i}}{L_{f i}} i_{f d i}-\omega_{i} i_{f q i}+\frac{1}{L_{f i}} v_{\text {Idrefi }}-\frac{1}{L_{f i}} v_{\text {odi }} \\
& \dot{v_{\text {odi }}}=\omega_{i} v_{o q i}+\frac{1}{C_{f i}} i_{f d i}-\frac{1}{C_{f i}} i_{\text {odi }} \\
& \dot{v_{\text {oqi }}}=-\omega_{i} v_{\text {odi }}+\frac{1}{C_{f i}} i_{f f i}-\frac{1}{C_{f i}} i_{o q i}
\end{aligned}
$$

where $\omega$ is the operating radian frequency which is determined by droop control (8), $r_{f}, L_{f}$, and $C_{f}$ are the resistance, inductance and capacitance of $L C$ filter respectively, $i_{f d}$ and $i_{f q}$ are the $d-q$ components of $L C$ filter inductance currents, respectively, $v_{o d}$ and $v_{o q}$ are $d-q$ components of the output voltage of DG unit at its related bus, respectively, $i_{o d}$ and $i_{o q}$ are $d-q$ components of DG unit output current, and $v_{I d, \text { ref }} \& v_{\text {Iq, ref }}$ are $d-q$ components of reference voltage which are input to the converter. The state space model is given as:

$$
\begin{aligned}
\dot{x}_{L C f i}= & A_{L C f i} x_{L C f i}+A_{L C f i, D r p i} x_{D r p i}+A_{L C f i, V S I i} x_{V S I i} \\
& +A_{L C f i, L D i} x_{L D i}+B_{L C f i, P N} u_{L C f i, P N}+B_{L C f i} u_{L C f i}
\end{aligned}
$$

where $\quad x_{L C f i}=\left[\begin{array}{llll}\Delta i_{f d i} & \Delta i_{f q i} & \Delta v_{o d i} & \Delta v_{o q i}\end{array}\right]^{T}$

$A_{L C f}=\left[\begin{array}{cccc}\frac{-r_{f i}-k_{p c i}}{L_{f i}} & -k_{p i} P_{0 i} & \frac{1-k_{p v i} k_{p c i}}{L_{f}} & \frac{-\omega_{0} C_{f i} k_{p c i}}{L_{f}} \\ +k_{p i} P_{0 i} & \frac{-r_{f i}-k_{p c i}}{L_{f i}} & \frac{\omega_{0} C_{f i} k_{p c i}}{L_{f i}} & \frac{1-k_{p v i} k_{p c i}}{L_{f i}} \\ \frac{1}{C_{f i}} & 0 & 0 & \omega_{0}-k_{p i} P_{0 i} \\ 0 & \frac{1}{C_{f i}} & -\omega_{0}+k_{p i} P_{0 i} & 0 \\ {[} & & -k & k\end{array}\right]$

$A_{L C f, D r p}=\left[\begin{array}{ccc}0 & -k_{p i} i_{f q i 0} & \frac{-k_{p c i} k_{p v i} k_{q i}}{L_{f i}} \\ 0 & +k_{p i} i_{f d i 0} & 0 \\ 0 & -k_{p i} v_{o q i 0} & 0 \\ 0 & +k_{p i} v_{o d i 0} & 0\end{array}\right]$ 
$A_{L C f, V S I}=\left[\begin{array}{cccc}\frac{k_{p c i} k_{l v i}}{L_{f i}} & 0 & \frac{k_{I c i}}{L_{f i}} & 0 \\ 0 & \frac{k_{p c i} k_{l v i}}{L_{f i}} & 0 & \frac{k_{I c i}}{L_{f i}} \\ 0 & 0 & 0 & 0 \\ 0 & 0 & 0 & 0\end{array}\right]$

$A_{L C f, L D i}=B_{L C f, P N}=B_{L C i i}=\left[\begin{array}{ccccc}\frac{F_{i} k_{p c i}-k_{p c i} k_{p v i} R_{v i}}{L_{f i}} & \frac{-k_{p c i} k_{p v i} \omega_{0} L_{v i}}{L_{f i}} & \frac{-1}{C_{f i}} & 0 \\ \frac{k_{p c i} k_{p v i} \omega_{0} L_{v i}}{L_{f i}} & \frac{F_{i} k_{p c i}-k_{p c i} k_{p v i} R_{v i}}{L_{f i}} & 0 & \frac{-1}{C_{f i}}\end{array}\right]^{T}$

$u_{L C f, P N}=\left[\sum_{j=1}^{n} \Gamma_{i j} \Delta i_{i j d} \sum_{j=1}^{n} \Gamma_{i j} \Delta i_{i j q} \mid \quad u_{L C f i}=\left[\begin{array}{ll}\Delta i_{L D C o n, i d} & \Delta i_{L D C o n, i q}\end{array}\right]^{T}\right.$

5) Local Load: it consists of $R L$ and constant loads:

$R L$ load: the dynamic model of the $R L$ load branch is obtained as:

$$
\begin{aligned}
& \dot{i_{L D d i}}=\frac{-R_{L D i}}{L_{L D i}} i_{L D d i}+\omega_{i} i_{L D q i}+\frac{1}{L_{L D i}} v_{o d i} \\
& \dot{i_{L D q i}}=\frac{-R_{L D i}}{L_{L D i}} i_{L D q i}-\omega_{i} i_{L D d i}+\frac{1}{L_{L D i}} v_{o q i}
\end{aligned}
$$

where $R_{L D i}, L_{L D i}$ and $i_{L D i}$ are resistance, inductance and current of the $R L$ load branch, and $v_{\text {odi }} \& v_{\text {oqi }}$ are $d-q$ components of the bus voltage magnitude. The state space model of load current in $d-q$ reference frame is obtained as:

$\dot{x}_{L D i}=A_{L D i} x_{L D i}+A_{L D i, D r p i} x_{D r p i}+A_{L D i, L C i} x_{L C f i}$

where $\quad x_{L D i}=\left[\begin{array}{ll}\Delta i_{L D d i} & \Delta i_{L D q i}\end{array}\right]^{T}$

$A_{L D i}=\left[\begin{array}{cc}\frac{-R_{L D i}}{L_{L D i}} & \omega_{0}-k_{p i} P_{0 i} \\ -\omega_{0}+k_{p i} P_{0 i} & \frac{-R_{L D i}}{L_{L D i}}\end{array}\right]$

$A_{L D i, L C i}=\left[\begin{array}{cccc}0 & 0 & \frac{1}{L_{L D i}} & 0 \\ 0 & 0 & 0 & \frac{1}{L_{L D i}}\end{array}\right] \quad A_{L D i, D r p i}=\left[\begin{array}{ccc}0 & -k_{p i} i_{L D d i 0} & 0 \\ 0 & +k_{p i} i_{L D q i 0} & 0\end{array}\right]$

In order to obtain small signal model of local $R L$ load in $P$ and $Q$ form, active and reactive power of load are obtained as:

$\left[\begin{array}{c}P_{L D i} \\ Q_{L D i}\end{array}\right]=\left[\begin{array}{cc}v_{\text {odi }} & v_{\text {oqi }} \\ v_{\text {oqi }} & -v_{\text {odi }}\end{array}\right]\left[\begin{array}{c}i_{L D d i} \\ i_{L D q i}\end{array}\right]$

The small signal model of load $(P \& Q)$ is obtained as:

$\left[\begin{array}{ll}\Delta P_{L D i} & \Delta Q_{L D i}\end{array}\right]^{T}=H_{L D i, L C f i} x_{L C f i}+H_{L D i, L D i} x_{L D i}$

where $\quad H_{L D i, L D i}=\left[\begin{array}{cc}v_{\text {odi }} & v_{\text {oqi } 0} \\ v_{\text {oqi } 0} & -v_{\text {odi } 0}\end{array}\right]$

$H_{L D i, L C f i}=\left[\begin{array}{cccc}0 & 0 & i_{L D d i 0} & i_{L D q i 0} \\ 0 & 0 & -i_{L D q i 0} & i_{L D d i 0}\end{array}\right]$

Constant load: $P$ and $Q$ related to the constant load is obtained as:

$\left[\begin{array}{c}P_{L D c o n i} \\ Q_{L D C o n i}\end{array}\right]=\left[\begin{array}{cc}v_{o d i} & v_{o q i} \\ v_{o q i} & -v_{o d i}\end{array}\right]\left[\begin{array}{c}i_{L D C o n, d i} \\ i_{L D C o n, q i}\end{array}\right]$

Accordingly, the small signal model of the constant load $(P \& Q)$ is obtained as:
$\left[\begin{array}{c}\Delta P_{L D C o n i} \\ \Delta Q_{L D C o n i}\end{array}\right]=H_{L D C o n i, L C f i} x_{L C f i}+H_{L D C o n i, L D C o n i} u_{L D C o n i}$

where $H_{L D C o n i, L C f i}=\left[\begin{array}{cccc}0 & 0 & i_{L D C o n, d i 0} & i_{L D C o n, q i 0} \\ 0 & 0 & -i_{L D C o n, q i 0} & i_{L D C o n, d i 0}\end{array}\right]$

$H_{L D C o n i, L D C o n i}=\left[\begin{array}{cc}v_{\text {odi } 0} & v_{\text {oqi } 0} \\ v_{\text {oqi } 0} & -v_{\text {odi } 0}\end{array}\right] \quad u_{L D C o n i}=\left[\begin{array}{c}i_{L D C o n, d i 0} \\ i_{L D C o n, q i 0}\end{array}\right]$

Then (26) is updated by (41) and (43).

\section{B. Load Buses}

Load bus includes its corresponding local load (where the dynamic model of load is obtained similarly to the local load model in Section IV.A) and shunt capacitor for power factor improvement. The state variable of the load bus is modeled as:

$\dot{v}_{L B d i}=\omega_{0} v_{L B q i}-\frac{1}{C_{C i}} \sum_{j=1}^{n} \Gamma_{i j} i_{i j d}-\frac{1}{C_{C i}} i_{L D d i}-\frac{1}{C_{C i}} i_{L D C o n d i}$

$\dot{v}_{L B q i}=-\omega_{0} v_{L B d i}-\frac{1}{C_{C i}} \sum_{j=1}^{n} \Gamma_{i j} i_{i j q}-\frac{1}{C_{C i}} i_{L D q i}-\frac{1}{C_{C i}} i_{L D C o n q i}$

where $i=k+1, \ldots, n$ and $C_{c i}$ is the capacitance of the shunt compensator. Consequently, the state space model is represented as:

$\dot{x}_{L B i}=A_{L B i} x_{L B i}+A_{L B i, L D i} x_{L D i}+B_{L B i, P N} u_{L B i, P N}+B_{L B i} u_{L B i}$

where $\quad x_{L B i}=\left[\begin{array}{ll}\Delta v_{L B d i} & \Delta v_{L B q i}\end{array}\right]^{T}$

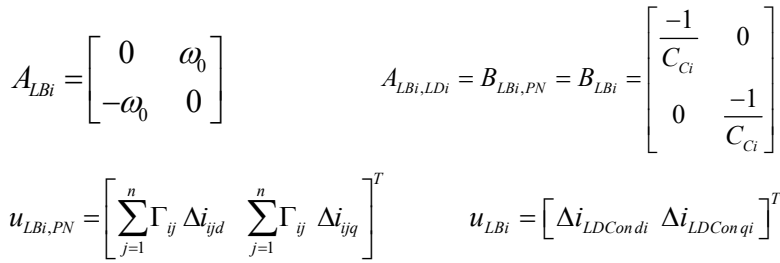

The dynamic model of phase angle $(\Delta \delta)$ at load bus is obtained as:

$\delta_{L B}=\arctan \left(v_{L B q} / v_{L B d}\right)$

Adopting (44)-(47) the voltage magnitude and phase angle dynamics at load bus are determined by currents dynamics flowing from adjacent buses. The currents dynamics, in turn, are determined by droop controller at adjacent nodes via (15). As a result, interaction of droop controller is realized at load buses, which is applicable to MG's main bus as well. The complete state space model of each load bus is obtained as (combining capacitor and load models):

$\dot{x}_{L B C i}=A_{L B C i i} x_{L B C i}+B_{L B C i, P N} u_{L B C i, P N}+B_{L B C i} u_{L B C i}$

where $\quad x_{L B C i}=\left[\begin{array}{ll}x_{L B i}^{T} & x_{L D i}^{T}\end{array}\right]^{T}$

$A_{L B C i}=\left[\begin{array}{cc}A_{L B i} & A_{L B i, L D i} \\ A_{L D i, L B i} & A_{L D i}\end{array}\right], A_{L D i, L B i}=\left[\begin{array}{cc}\frac{1}{L_{L D i}} & 0 \\ 0 & \frac{1}{L_{L D i}}\end{array}\right]$

$B_{L B C i, P N}=\left[\begin{array}{ll}B_{L B i, P N}^{T} & 0_{2 \times 2}\end{array}\right]^{T}, B_{L B C i}=\left[\begin{array}{cc}B_{L B i}^{T} & 0_{2 \times 2}\end{array}\right]^{T}$

$u_{L B C i, P N}=\left[\sum_{j=1}^{n} \Gamma_{i j} \Delta i_{i j d} \sum_{j=1}^{n} \Gamma_{i j} \Delta i_{i j q}\right]^{T} \quad u_{L B C i}=\left[\Delta i_{L D C o n d i} \Delta i_{L D C o n q i}\right]^{T}$ 
$A_{L D i}$ is similar in (39) except that $k_{p}$ (the droop gain) in a load bus is zero.

\section{Power Network}

The dynamic model of current flowing through the $i j^{\text {th }}$ power line (15) is obtained as:

$$
\begin{aligned}
& {\left[\begin{array}{cc}
\Delta i_{i j d} & \Delta i_{i j q}
\end{array}\right]^{T}=H_{i_{i j}, D r p i} x_{D r p i}+H_{i_{i j}, D r p j} x_{D r p j}} \\
& +H_{i_{i j}, L C f i} x_{L C f i}+H_{i_{i j}, L C f j} x_{L C f j} \\
& +H_{i_{i j}, L B i} x_{L B i}+H_{i_{i j}, L B j} x_{L B j} \\
& \text { where } H_{i_{i j}, D r p i}=\left[\begin{array}{lll}
\frac{\partial i_{i j d}}{\partial \delta_{i}} & 0 & 0 \\
\frac{\partial i_{i j q}}{\partial \delta_{i}} & 0 & 0
\end{array}\right], H_{i_{i j}, D r p j}=\left[\begin{array}{lll}
\frac{\partial i_{i j d}}{\partial \delta_{j}} & 0 & 0 \\
\frac{\partial i_{i j q}}{\partial \delta_{j}} & 0 & 0
\end{array}\right] \\
& H_{i_{i j}, L C f i}=\left[\begin{array}{cccc}
0 & 0 & \frac{\partial i_{i j d}}{\partial v_{o d i}} & \frac{\partial i_{i j d}}{\partial v_{o q i}} \\
0 & 0 & \frac{\partial i_{i j q}}{\partial v_{o d i}} & \frac{\partial i_{i j q}}{\partial v_{o q i}}
\end{array}\right], H_{i_{i j}, L C f, j}=\left[\begin{array}{cccc}
0 & 0 & \frac{\partial i_{i j d}}{\partial v_{o d j}} & \frac{\partial i_{i j d}}{\partial v_{o q j}} \\
0 & 0 & \frac{\partial i_{i j q}}{\partial v_{o d j}} & \frac{\partial i_{i j q}}{\partial v_{o q j}}
\end{array}\right] \\
& H_{i_{i j}, L B i}=\left[\begin{array}{cc}
\frac{\partial i_{i j d}}{\partial v_{L B d i}} & \frac{\partial i_{i j d}}{\partial v_{L B q i}} \\
\frac{\partial i_{i j q}}{\partial v_{L B d i}} & \frac{\partial i_{i j q}}{\partial v_{L B q i}}
\end{array}\right], H_{i_{i j}, L B j}=\left[\begin{array}{cc}
\frac{\partial i_{i j d}}{\partial v_{L B d j}} & \frac{\partial i_{i j d}}{\partial v_{L B q j}} \\
\frac{\partial i_{i j q}}{\partial v_{L B d j}} & \frac{\partial i_{i j q}}{\partial v_{L B q j}}
\end{array}\right]
\end{aligned}
$$

The dynamic model of $P$ and $Q$ flowing through the $i j^{\text {th }}$ power line (17) is obtained as:

$$
\begin{aligned}
& {\left[\begin{array}{ll}
P_{i j} & Q_{i j}
\end{array}\right]^{T}=H_{P Q_{i j}, D r p i} x_{D r p i}+H_{P Q_{i j}, D r p j} x_{D r p j}} \\
& +H_{P Q_{i j}, L C f i} x_{L C f i}+H_{P Q_{i j}, L C f j} x_{L C f j} \\
& +H_{P Q_{i j}, L B i} x_{L B i}+H_{P Q_{i j}, L B j} x_{L B j} \\
& \text { where } H_{P Q_{i j}, D r p i}=\left[\begin{array}{ccc}
\frac{\partial P_{i j}}{\partial \delta_{i}} & 0 & 0 \\
\frac{\partial Q_{i j}}{\partial \delta_{i}} & 0 & 0
\end{array}\right], H_{P Q_{i j}, D r p j}=\left[\begin{array}{ccc}
\frac{\partial P_{i j}}{\partial \delta_{j}} & 0 & 0 \\
\frac{\partial Q_{i j}}{\partial \delta_{j}} & 0 & 0
\end{array}\right] \\
& H_{P Q_{i j}, L C f, i}=\left[\begin{array}{cccc}
0 & 0 & \frac{\partial P_{i j}}{\partial v_{o d i}} & \frac{\partial P_{i j}}{\partial v_{o q i}} \\
0 & 0 & \frac{\partial Q_{i j}}{\partial v_{o d i}} & \frac{\partial Q_{i j}}{\partial v_{o q i}}
\end{array}\right], H_{P Q_{i j}, L C f, j}=\left[\begin{array}{cccc}
0 & 0 & \frac{\partial P_{i j}}{\partial v_{o d j}} & \frac{\partial P_{i j}}{\partial v_{o q j}} \\
0 & 0 & \frac{\partial Q_{i j}}{\partial v_{o d j}} & \frac{\partial Q_{i j}}{\partial v_{o q j}}
\end{array}\right] \\
& H_{P Q_{i j}, L B i}=\left[\begin{array}{ll}
\frac{\partial P_{i j}}{\partial v_{L B d i}} & \frac{\partial P_{i j}}{\partial v_{L B q i}} \\
\frac{\partial Q_{i j}}{\partial v_{L B d i}} & \frac{\partial Q_{i j}}{\partial v_{L B q i}}
\end{array}\right], H_{P Q_{i j}, L B j}=\left[\begin{array}{cc}
\frac{\partial P_{i j}}{\partial v_{L B d j}} & \frac{\partial P_{i j}}{\partial v_{L B q j}} \\
\frac{\partial Q_{i j}}{\partial v_{L B d j}} & \frac{\partial Q_{i j}}{\partial v_{L B q j}}
\end{array}\right]
\end{aligned}
$$

Equations (49)-(50) model all branches of the power network in a same way. In order to distinguish those branches which connect generation nodes to passive nodes or two passive nodes, the following item should be taken into account. The models of flowing current (49) and active and reactive power (50) through a given power line are modified according to the power line type:

i) power line connecting two DG units: $(1 \leq i, j \leq k)$

$$
H_{i_{i, j}, L B i}=H_{i_{i j}, L B j}=H_{P Q_{i j}, L B i}=H_{P Q_{i j}, L B j}=0
$$

ii) power line connecting DG $i$ and load bus $j$ : $(1 \leq i \leq k, k+1 \leq j$ $\leq n)$
$H_{i_{i j}, D r p j}=H_{P Q_{i j}, D r p j}=0, H_{i i j}, L C f j=H_{P Q_{i j}, L C f j}=0, H_{i_{i j}, L B i}=H_{P Q_{i j}, L B i}=0$ iii) power line connecting load bus $i$ and DG $j:(k+1 \leq i \leq n, 1 \leq$ $j \leq k$ )

$H_{i_{i j}, D r p i}=H_{P Q_{i j}, D r p i}=0, H_{i_{i j}, L C f i}=H_{P Q_{i j}, L C f i}=0, H_{i_{i j}, L B j}=H_{P Q_{i j}, L B j}=0$

iv) power line connecting load bus $i$ and load bus $j:(k+1 \leq i, j$ $\leq n)$

$$
\begin{aligned}
& H_{i_{i j}, D r p i}=H_{i_{i j}, D r p j}=0, H_{i_{i j}}, L C f i=H_{i j}, L C f j=0 \\
& H_{P Q_{i j}, D r p i}=H_{P Q_{i j}, D r p j}=0, H_{P Q_{i j}, L C f i}=H_{P Q_{i j}}, L C f j=0
\end{aligned}
$$

$\boldsymbol{R} \boldsymbol{F}$ at power network: the power network can be categorized into the following parts:

1. Generation buses: the RF of generation buses is specified by droop controllers.

2. Load buses: the RF of load buses is determined using $d-q$ component of voltage at these buses as presented in Section IV.

3. Connecting buses: the RF of connecting buses (buses with no generation units nor loads which connect more than two lines) can be determined with a process similar to load buses by assuming a virtual capacitor of a very small capacitance at connecting buses so that the capacitor current is negligible in comparison to the network nominal current. However, it does not significantly influence the dynamic stability of MG since the RF at connecting buses depends on RF of generation buses.

4. Power lines: power lines are modelled by its impedance including resistance and reactance. The frequency variation at power network nodes affects the power line models by changing the line reactance. However, the frequency variation is not too much to be effective in dynamic stability studies. To investigate this issue, let take the well-known power transfer equation in an inductive line:

$\Delta P_{i j} \approx \frac{|V|^{2}}{X_{i j}} \Delta \delta_{i j}$

where $\frac{\partial P_{i j}}{\partial f} \approx-\frac{2 \pi L|V|^{2}}{X_{i j}^{2}} \delta_{i j}$ which is a small value compared with $\frac{\partial P_{i j}}{\partial \delta_{i j}} \approx \frac{|V|^{2}}{X_{i j}}$. Hence, phase angle dynamics, as the consequence of frequency variation, is an important factor in dynamic modelling of NMGs affecting the RF at generation nodes. While, the reactance variation of power line as the consequence of frequency variation is not an effective factor.

\section{Whole NMG Model}

After inserting power network model into the model of each component, the state space model of whole NMG is obtained as:

$$
\begin{aligned}
\dot{x}_{M G} & =A_{M G} x_{M G}+B_{M G} u_{M G} \\
x_{M G} & =\left[\begin{array}{lllllllll}
x_{D G 1}^{T} \ldots & x_{D G i}^{T} & \ldots & x_{D G k}^{T} & x_{L B C k+1}^{T} \ldots & x_{L B C k+h}^{T} & \ldots & x_{L B C n}^{T}
\end{array}\right]^{T} \\
B_{M G} & =\operatorname{diag}\left[\begin{array}{llllllll}
B_{D G 1} \ldots & B_{D G i} & \ldots & B_{D G k} & B_{L B C k+1} & \ldots & B_{L B C k+h} \ldots & B_{L B C n}
\end{array}\right]
\end{aligned}
$$




$$
\begin{aligned}
& B_{D G i}=\left[\begin{array}{lllll}
0_{2 \times 2} & \left(B_{D r p i, L C c o n i} H_{L D C o n i}{ }_{L D C O n i}\right)^{T} & B_{V S C i}^{T} & B_{L C f i}^{T} & 0_{2 \times 2}
\end{array}\right]^{T} \\
& u_{M G}=\left[\begin{array}{lll}
u_{D G 1}^{T} \ldots u_{D G i}^{T} \ldots u_{D G k}^{T} & u_{L B C k+1}^{T} \ldots u_{L B C k+h}^{T} \ldots u_{L B C h}^{T}
\end{array}\right]^{T} \\
& u_{D G i}=u_{L B C i}=\left[\begin{array}{lll}
\Delta i_{L D C o n, d i} & \Delta i_{L D C o n, q i}
\end{array}\right]^{T}
\end{aligned}
$$

$A_{M G}$ is presented in the Appendix.

\section{Simulations AND Eigenvalue Studies}

MATLAB code is used to draw the eigenvalue loci of the developed model to evaluate its effectiveness. Simulations are conducted in Simulink/Simscape, Power Systems toolbox to obtain the variable values at the operating points and to validate the model. The simulated MG is shown in Fig. 6. The VSI control system which is adopted for developing the Simulink model is represented in Fig. A1 in the Appendix. The nominal frequency is $60 \mathrm{~Hz}$, the nominal Phase-to-Phase voltage is 400 $\mathrm{V}$ and the demanded active and reactive power representing the common load (CL) in Fig. 6 are $20 \mathrm{~kW}$ and 10 kvar, respectively. The demanded power is distributed among the VSIs according to the assigned droop gains represented in Fig. 6 . The reactive power, however, is not dispersed according to the droop gains, which is the main shortcoming of the $V-Q$ droop loop in reactive sharing. This issue is studied in the literature [8], [27] and is beyond the scope of this paper.

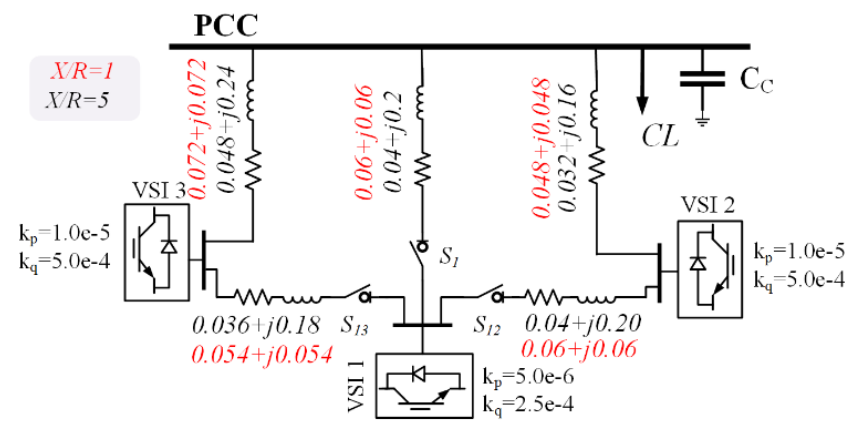

Fig. 6. Schematic diagram of the simulated microgrid; for meshed (networked) topology $S_{1}$ is open, $S_{12}$ and $S_{13}$ are closed; for parallel topology $\mathrm{S}_{1}$ is closed, $\mathrm{S}_{12}$ and $\mathrm{S}_{13}$ are open.

\section{A. Model Validation}

In this section, accuracy of the developed small-signal model as well as the Simulink model are assessed using well-known facts, i.e. the higher $\mathrm{X} / \mathrm{R}$ ratio of power lines the lower cross coupling between $f-P$ and $V$ - $Q$ droop loops, and the larger droop gains the narrower stability margin. In order to identify critical dominant poles related to $f-P$ and $V$ - $Q$ droop loops, the droop gains and the $\mathrm{X} / \mathrm{R}$ ratio of power line impedance are increased to observe eigenvalues movements.

The dominant eigenvalues of the meshed MG in Fig. 6 are depicted in Figs. 7(a)-(e). Increasing droop gains pushes the corresponding poles to the right side (arrow direction) and recognizes two sets of complex-conjugate poles, which as it is investigated in the following, reveal critical oscillation modes. Mode 1 specifies a low frequency oscillation related to the $f-P$ droop loop and Mode 2 specifies a higher frequency oscillation related to the $V-Q$ droop loop. In Fig. 7, the eigenvalues indicated by a star "*” with black colour are related to the nominal droop gains given in Fig. 6. The simulation results corresponding to the eigen-analysis are shown in Figs. 8(a)-(f). The following items can be deduced from the study which are confirmed from both eigen-analysis and simulation results:
- When the $\mathrm{X} / \mathrm{R}=1$, the high cross-coupling of $f-P$ and $V$ - $Q$ droop loops as well as strong interaction of droop controllers makes the NMG unstable. It is shown by both the eigen loci and simulation result in Fig. 7(a) and Fig. $8(a)$, respectively, both with the nominal droop gains given in Fig. 6. In Fig. 7(a), the eigenvalues related to $f-P$ loop are located (originated) in the right-hand side of imaginary axis implying system instability. The frequency of unstable active power (frequency) oscillations given from the eigenvalue location in Fig. 7(a) is $20.1 \mathrm{rad} / \mathrm{s}$ and that one given from the time domain simulation in Fig. 8(a) is 22.2 $\mathrm{rad} / \mathrm{s}$, which are roughly same.

- With $\mathrm{X} / \mathrm{R}=1$, increasing the droop gain of $f-P(V-Q)$ loop has an inverse effect on $V-Q(f-P)$ loop, which realizes the cross-coupling effect. This can be observed in Fig. 7(a), as increasing $\mathrm{k}_{\mathrm{p}}\left(\mathrm{k}_{\mathrm{q}}\right)$ shifts corresponding poles of $V-Q(f-P)$ toward the left side. It is due to negative coupling of droop loops. To investigate this issue, consider the following equation which is a linearized version of the power flow equations with $\mathrm{X} / \mathrm{R}=1$ :

$\left[\begin{array}{c}\Delta P_{i j} \\ \Delta Q_{i j}\end{array}\right] \approx \frac{\sqrt{2}}{2} \frac{1}{Z_{i j}}\left[\begin{array}{cc}|V| & |V|^{2} \\ |V| & -|V|^{2}\end{array}\right]\left[\begin{array}{c}\Delta V_{i j} \\ \Delta \delta_{i j}\end{array}\right]$

Since, both $f-P$ and $V-Q$ loops are highly coupled and also, based on (52), the reactive power is negatively proportional to phase angle, the two loops are negatively coupled. This opposite effect comes from the high sensitivity of two droop loops to $\Delta \delta$, proportional to $|\mathrm{V}|^{2}$ but with opposite signs.

- With $\mathrm{X} / \mathrm{R}=1.5$, the control system is stable which is confirmed by both eigen loci and simulation result in Fig. 7(b) and Fig. 8(b), respectively. However, the two droop loops are still coupled so that both of them oscillate with approximately same frequency as shown in Fig. 8(c);

- With $\mathrm{X} / \mathrm{R}=5$, the droop loops are less coupled and the stability margins are improved. However, the crosscoupling between two droop loops is high enough to modulate high frequency oscillations of $V-Q$ loop into $f-P$ loop and vice versa. This can be observed in Fig. 7(c) and Fig. 8(d);

- The higher $\mathrm{X} / \mathrm{R}$ ratio results in a larger frequency of reactive power fluctuations with a smaller time constant, while it leads to a lower frequency of active power oscillation with a roughly fixed time constant dominated by the cut-off frequency of LPF; please refer to (11-b) in Section III.C for details.

- With $\mathrm{X} / \mathrm{R}=8$, both droop loops are decoupled, so that increasing droop gain of one droop loop does not affect the other droop loop as shown in Fig. 7(d). Fig. 8(e) also confirms this fact. Moreover, these two figures reveal that the larger $\mathrm{X} / \mathrm{R}$ leads to a higher damping factor which suppresses the overshoot value.

- Adopting virtual impedance decouples $f-P$ and $V-Q$ loops and improves the stability margins of NMG as illustrated in Fig. 7(e) through eigen-analysis and Fig. 8(f) via simulation results. Although the state variables related to the $d-q$ components of line currents are neglected in the model, the proposed model based on the developing current as a 
function of nodes' voltages properly reveals virtual impedance effect.
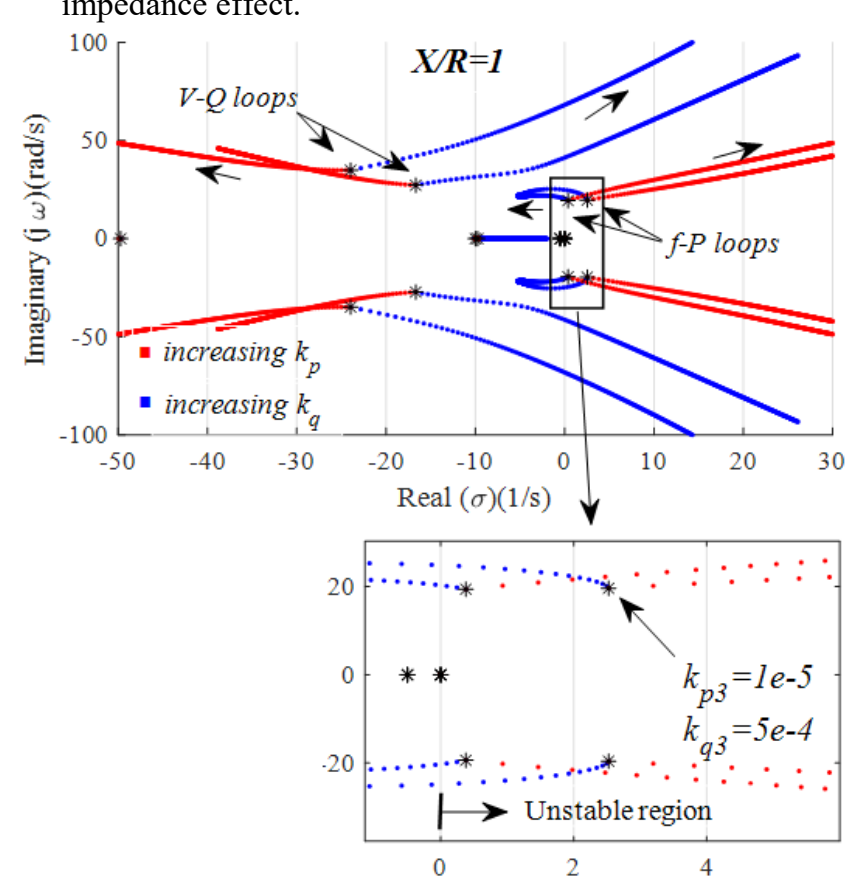

(a)
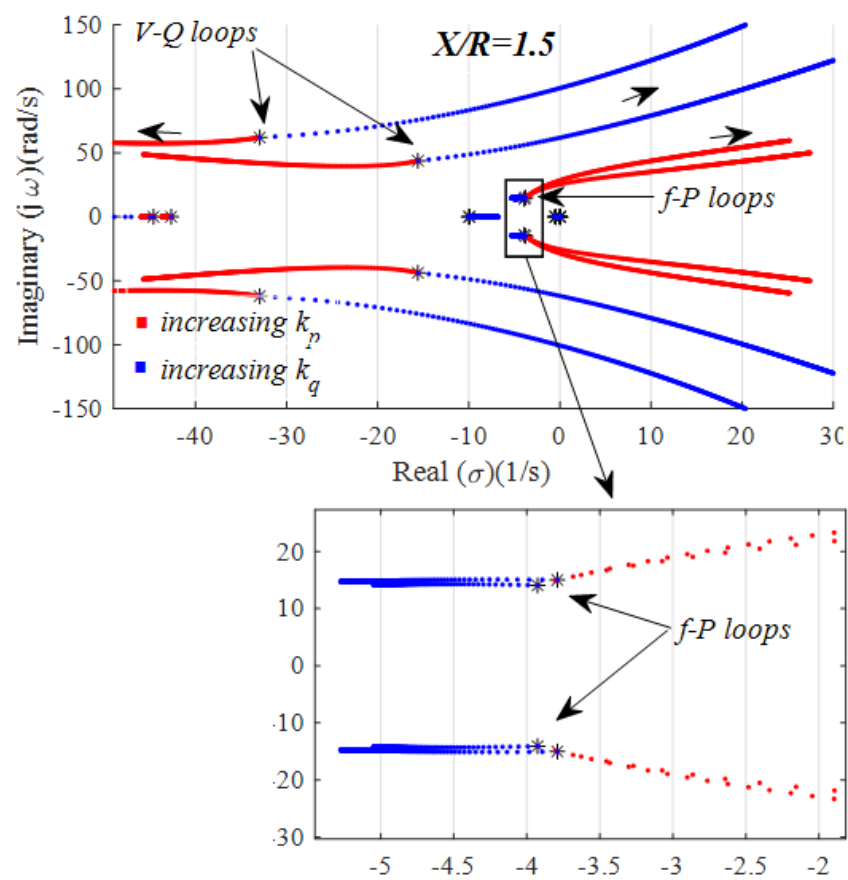

(b)
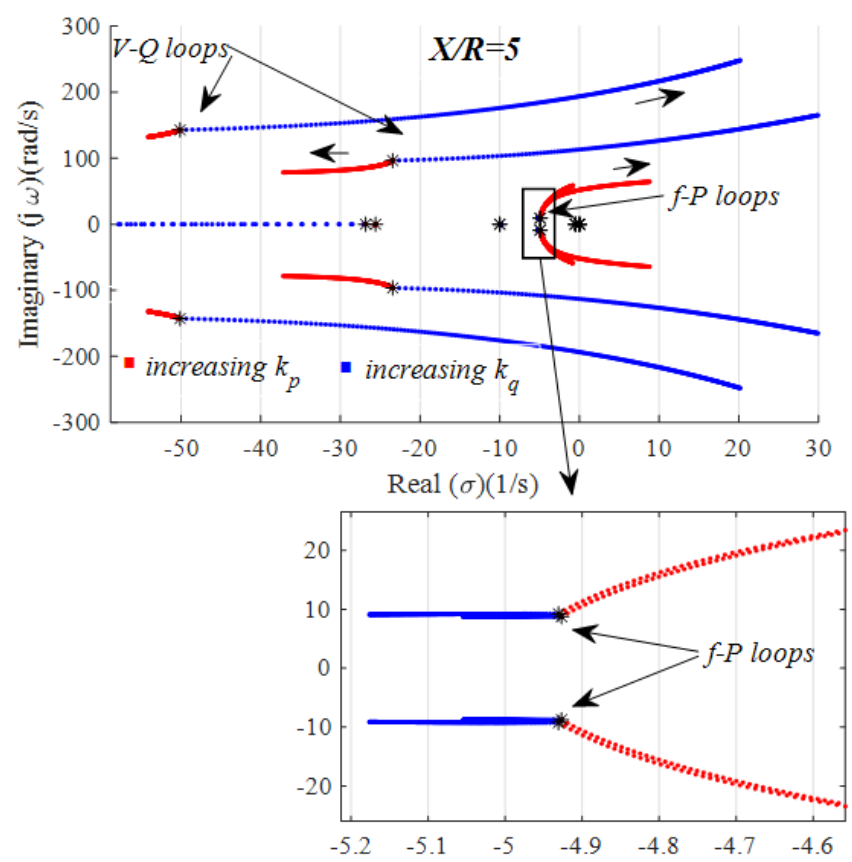

(c)
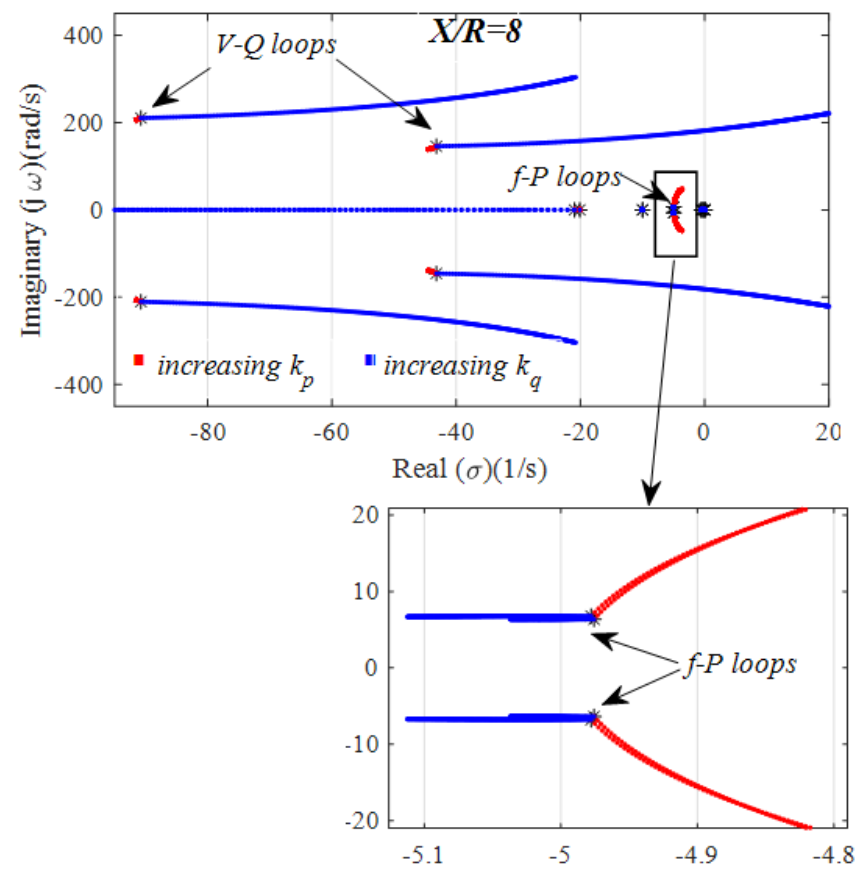

(d) 

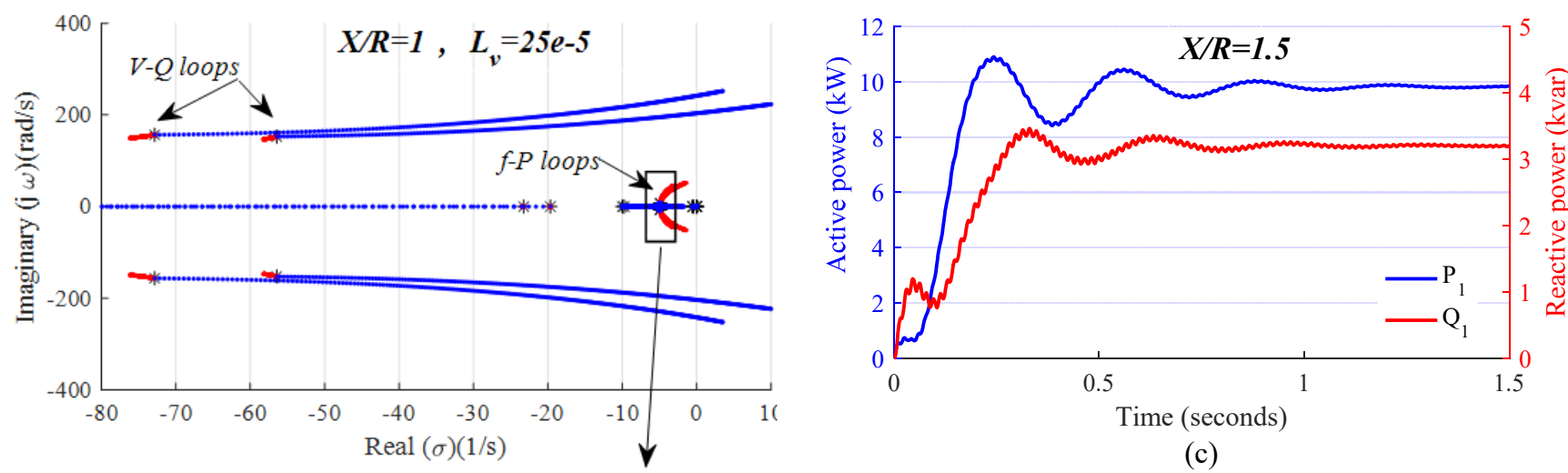

(c)

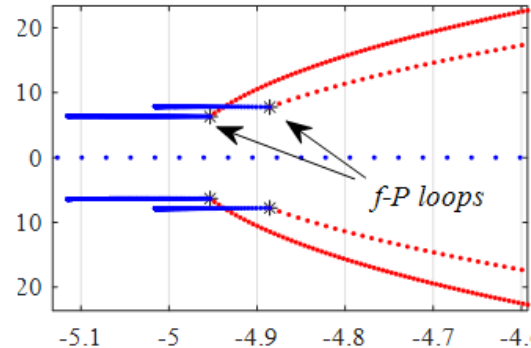

(e)

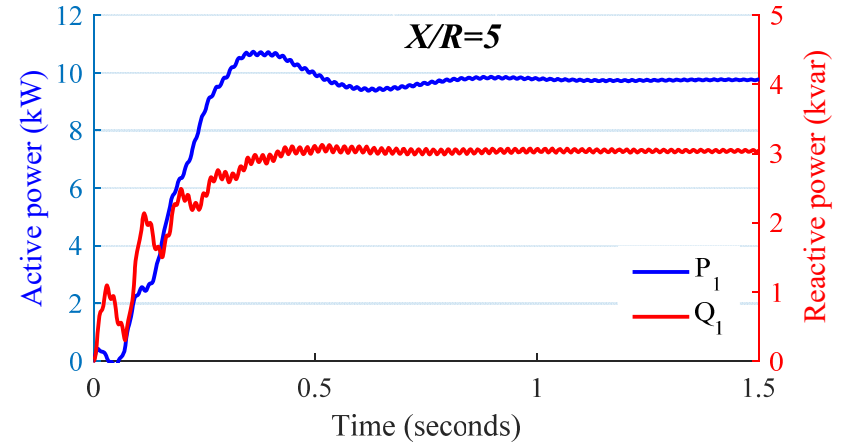

(d)

Fig. 7. Critical dominant poles for different $\mathrm{X} / \mathrm{R}$ ratios of the $\mathrm{NMG}$ (the eigenvalues indicated by a star "* " with black colour are related to the nominal droop gains given in Fig. 6.

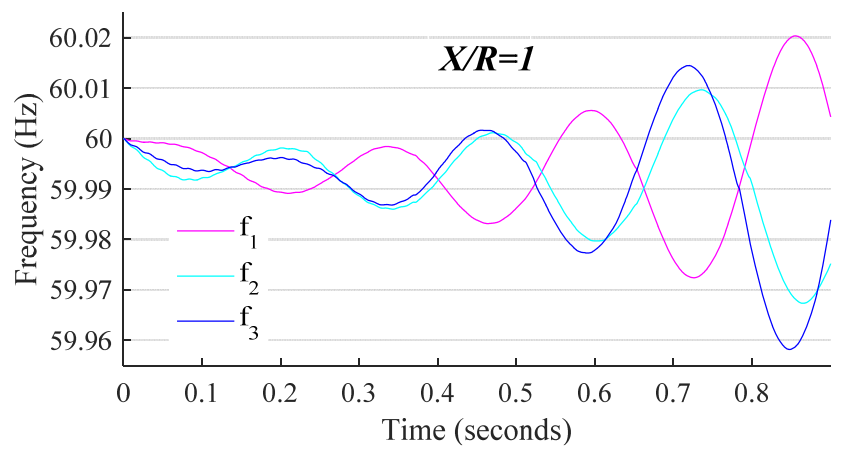

(a)

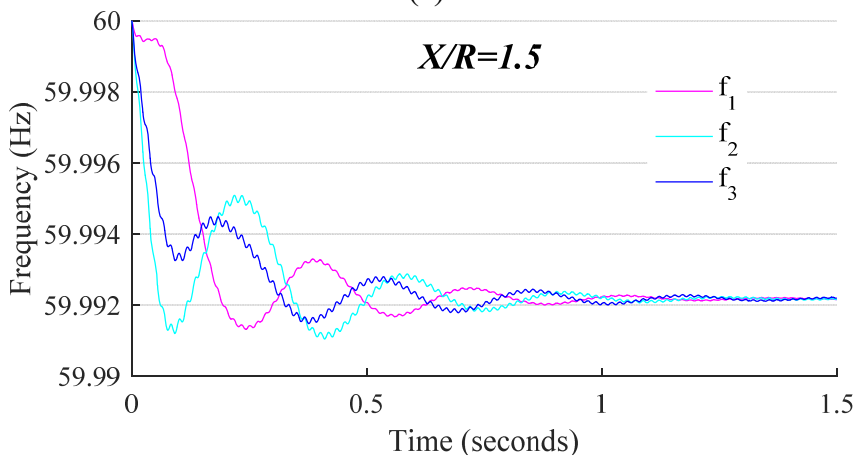

(b)

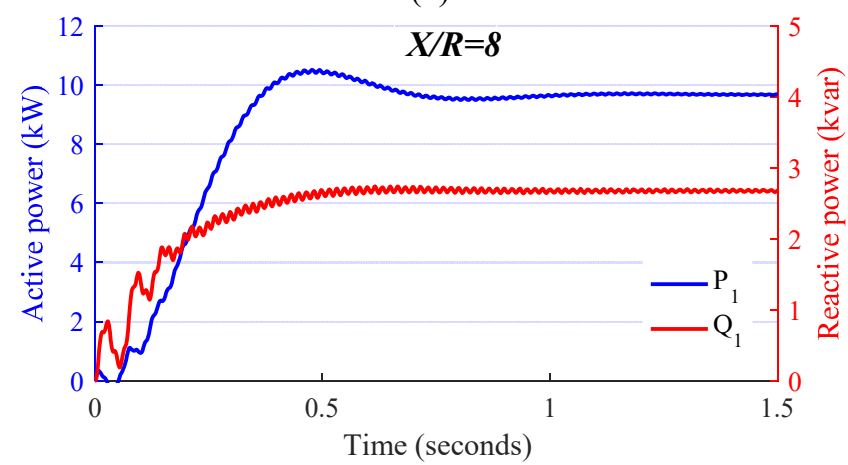

(e)

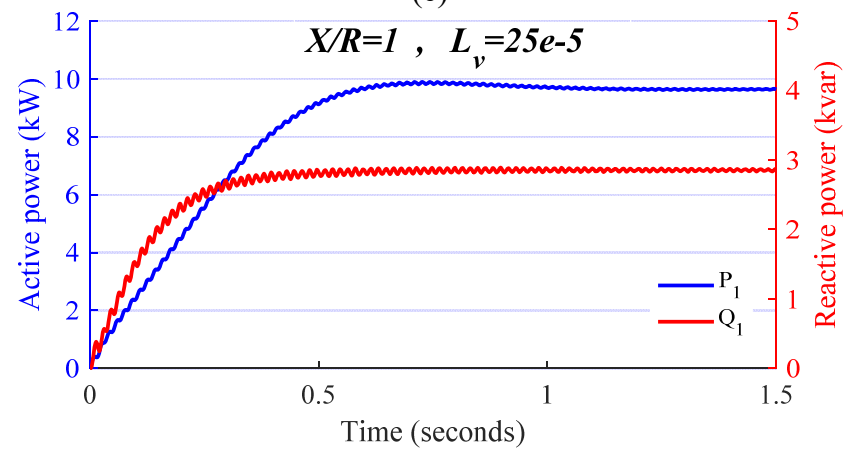

(f)

Fig. 8. (a)-(b) Simulation results, frequency dynamics of VSIs 1-3. (c)-(f) Simulation results, active power and reactive power dynamics of VSI 1.

\section{B. Comparison with PL-based model}

Since accuracy and sensitivity of the proposed NT-based model to power network parameters are evaluated in the previous subsection through the time domain simulation, here only a comparison between the NT-based model and the conventional model of parallel MGs (PL-based model [39]-[41]) is presented. Fig. 9(a) shows the dominant eigenvalue loci in relation to the 
variation of power network impedance. By decreasing the grid inductance, while the grid resistance is constant (which decreases the $X / R$ ratio as well), the system becomes unstable due to the cross-coupling effect between $f-P$ and $V-Q$ droop loops. The NT-based model represents the unstable regions, which the PL-based model fails to discover.

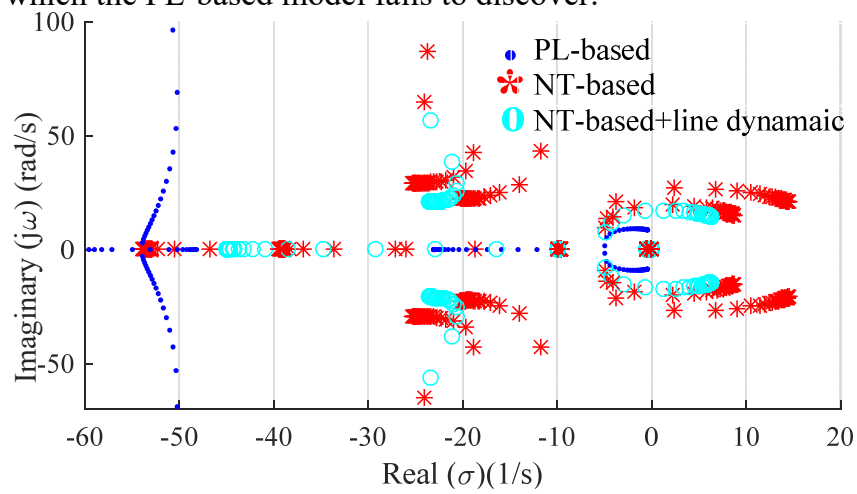

(a)

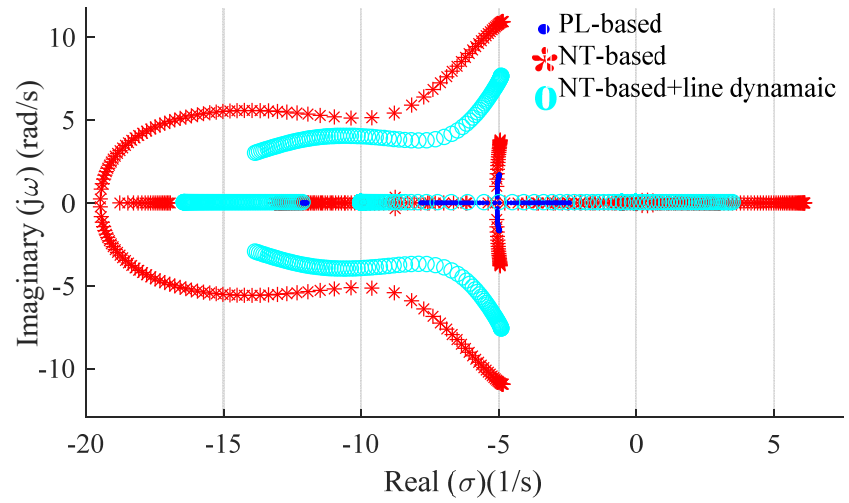

(b)

Fig. 9. Dominant eigenvalues loci, (a) in relation to grid impedance variation, (b) in relation to load increase (nominal point).

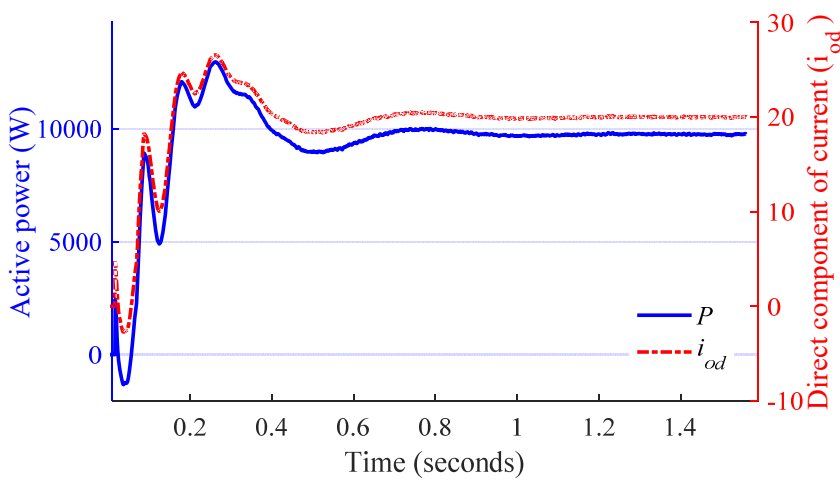

(a)

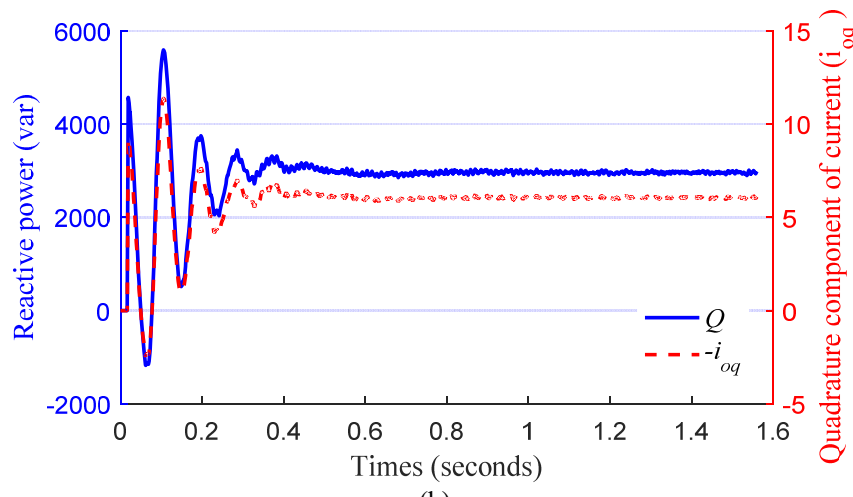

(b)

Fig. 10. (a) Active power and $i_{o d}$ dynamics of VSI 1. (b) Reactive power and $i_{o q}$ dynamics of VSI 1.

Fig. 9(b) reveals the unstable regions in relation to changes of the operating point (increase in the demand). Load raising leads to an increase of the phase angle difference between generation buses $\left(\Delta \delta_{i j}\right)$ and consequently stronger interaction among droop controllers due to high sensitivity of power to phase angle. It is shown that the droop-based MG becomes unstable in some operating points after increasing the load, while this is not disclosed by using the PL-based model. To have an accurate linearized model when the phase angle becomes large, the operating points like power, voltage and current values are updated when the load is increased.

Highlight3: In the proposed NT-based model, power network dynamics (electromagnetic transients) are ignored and this eliminates 2 states ( $d$ - $q$ components of the line current) per each interconnecting power line. This reduces the order of NMGs state-space model considerably. In order to assess the accuracy of the reduced-order model, the NT-based model including power lines dynamics, namely "NT-based + line dynamics", is developed and illustrated in Fig. 9 as well. Taking the line current as state variable makes Highlightl impractical, while Highlight2 is still applicable. Despite that the line dynamics is not inserted into the proposed NT-model, which reduces the model dimension considerably, the model is accurate in terms of dominant poles. Moreover, compatibility of the current fluctuations with power oscillations is represented in Fig. 10(a) for $i_{o d}$ with active power and in Fig. 10(b) for $i_{o q}$ with reactive power. This confirms that the current dynamics is affected by these two critical modes, while the feeder's electromagnetic transient, which is ignored in the small-signal model, is not an effective element.

\section{Interaction of droop controllers and power network topology}

In order to demonstrate the interaction of droop controllers, simulation results for a meshed MG is compared with a parallel MG consisting of three DG units. Fig. 11(a) indicates eigenvalue loci of the parallel $\mathrm{MG}$ which is stable for $\mathrm{X} / \mathrm{R}=1$. Figs. 11(b) and (c) show the simulation results for parallel MG with $X / R \approx 1$ which are in consistence with eigenvalue study. On the other hand, the control system in the meshed MG with the same $\mathrm{X} / \mathrm{R}$ ratio is unstable; as shown in Fig. 11(d). This is because of the interaction of droop controllers, which also makes the $f-P$ and $V$ - $Q$ droop loops highly coupled. The related eigenvalue loci given from the proposed model are depicted in Fig. 7(a), and show unstable modes related to the $f-P$ loops originated at the right-hand side of imaginary axis. In order to 
investigate the effect of the interaction of droop controllers, the relevant droop gains are considered zero making the control system stable, as seen in Figs. 11(d) and 11(e); the power sharing is lost though. In the next step, the $\mathrm{X} / \mathrm{R}$ ratio is considered a little bit higher $(X / R \approx 1.5)$. The eigenvalue loci in Fig. 7(b) reveal that the dominant modes are shifted to the lefthand side, which means that the system is stable. This is confirmed by the simulation results as shown in Figs. $11(\mathrm{~g})$ and $8(\mathrm{~h})$. The interaction of droop controllers, however, still makes the fluctuations of the system outputs too high in comparison to those in the parallel MG depicted in Figs. 8(b) and 8(c) even with the lower $X / R$ ratio.

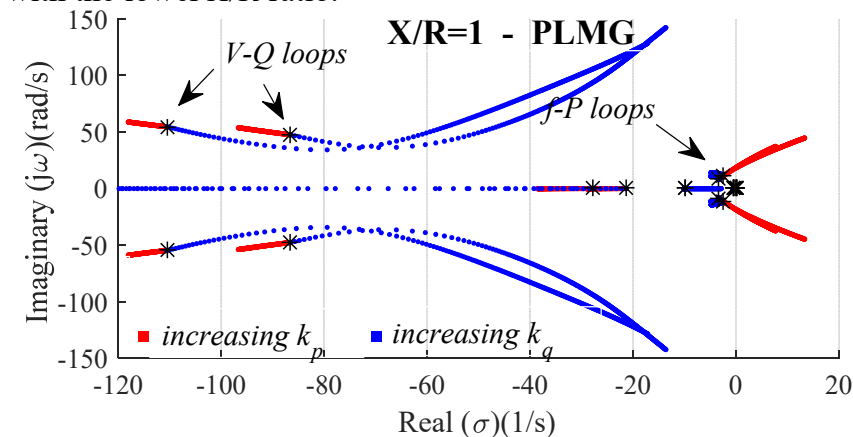

(a)

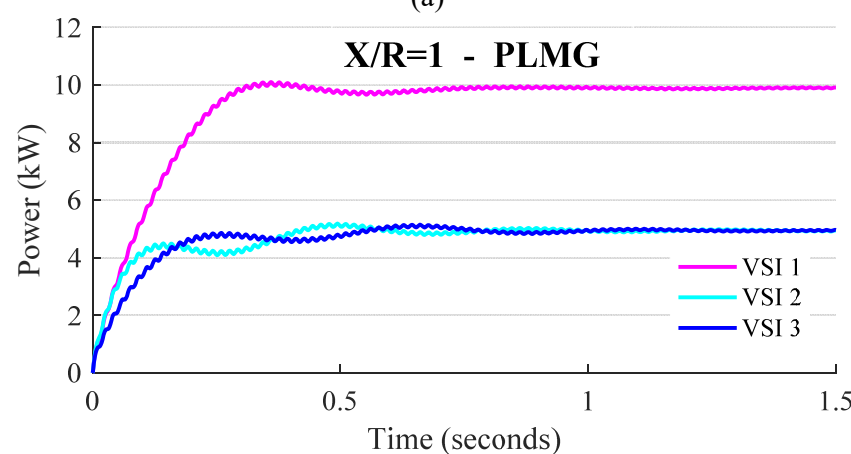

(b)

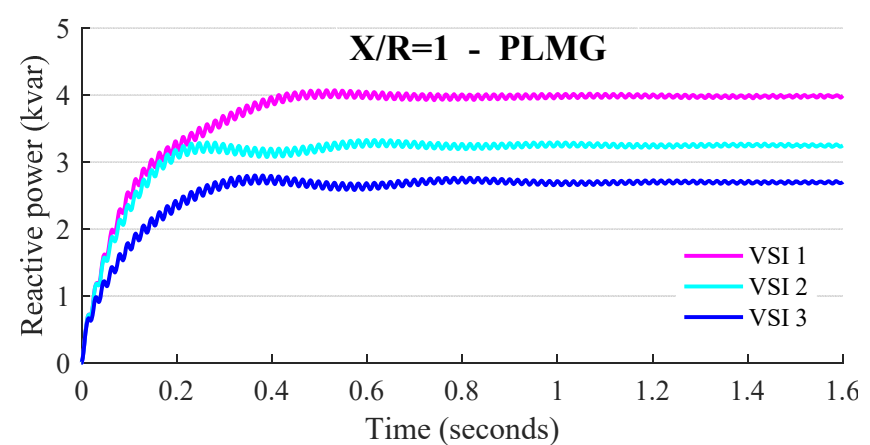

(c)

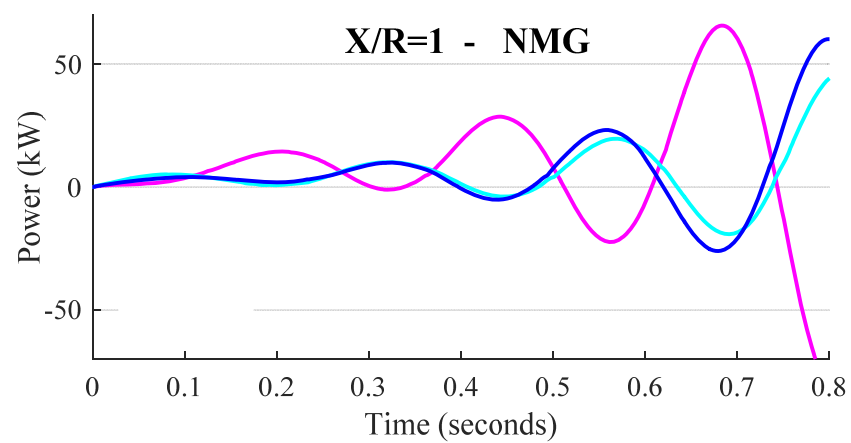

(d)

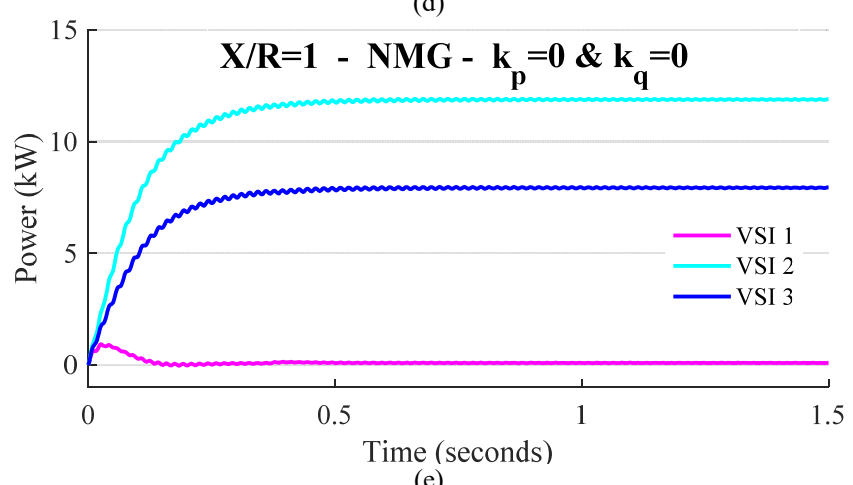

(e)

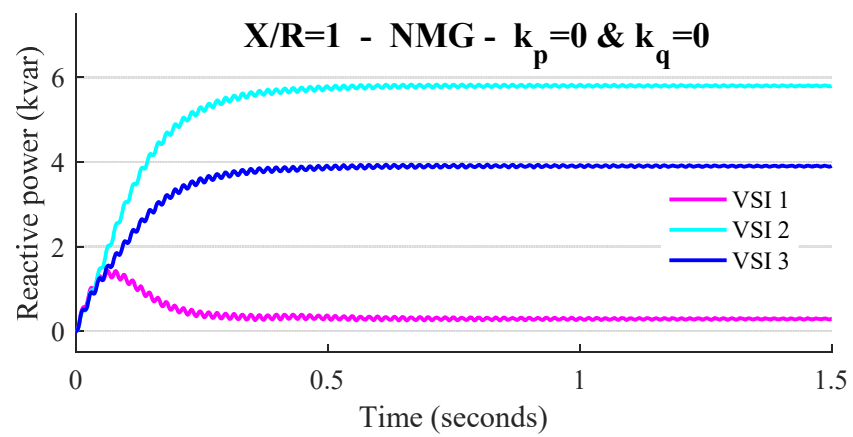

(f)

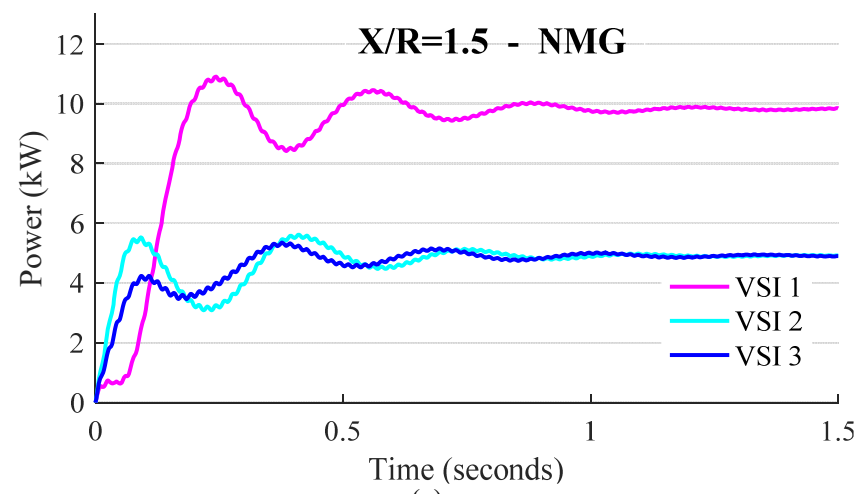

(g)

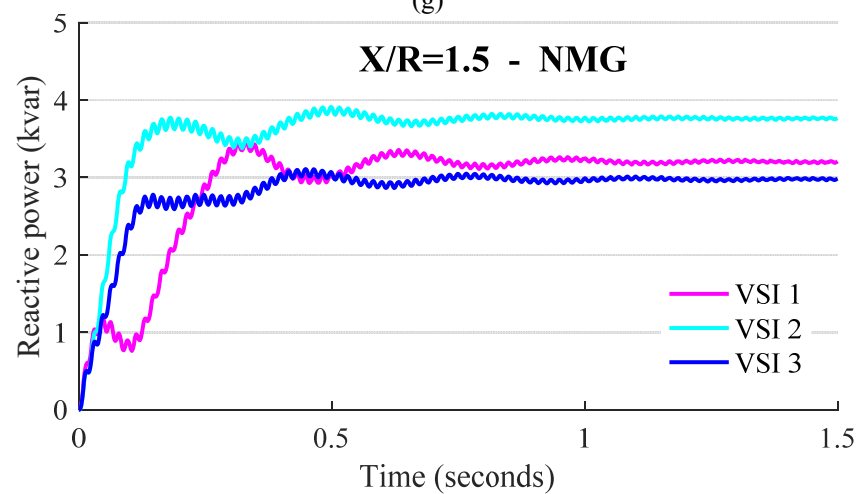

(h)

Fig. 11. (a) Eigen value loci of the Parallel MG (PLMG). (b)-(h) Simulation results for comparing dynamic response of the droop control in parallel and networked MGs with different $X / R$ ratio. (b)-(c) Parallel MG (X/R $\approx 1)$. (d) Networked MG (NMG) $(\mathrm{X} / \mathrm{R} \approx 1)$. (e), (f) Networked $M G\left(\mathrm{X} / \mathrm{R} \approx 1, k_{p}=0, k_{q}=0\right)$. (g), (h) Networked $M G(X / R \approx 1.5)$. 


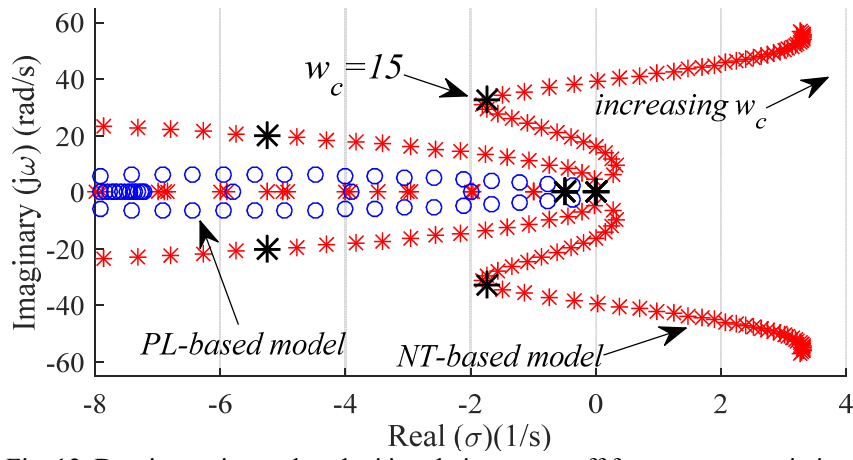

Fig. 12. Dominant eigenvalues loci in relation to cut-off frequency $\omega_{\mathrm{c}}$ variation.

\section{First-order filter}

The eigenvalue loci of the control system in relation to the cutoff frequency variation of LPF are shown in Fig. $12(X / R \approx 1)$. The NT-based model reveals some unstable regions which are not identified by the PL-based model. In addition, the cut-off frequency of $15 \mathrm{rad} / \mathrm{s}$ yields the best dynamic performance which is verified by simulation tests shown in Fig. 13. Moreover, as per the simulation and eigen-analysis, the larger cut-off frequency $\omega_{c}$, the higher oscillation frequency of the dominant modes in MGs.

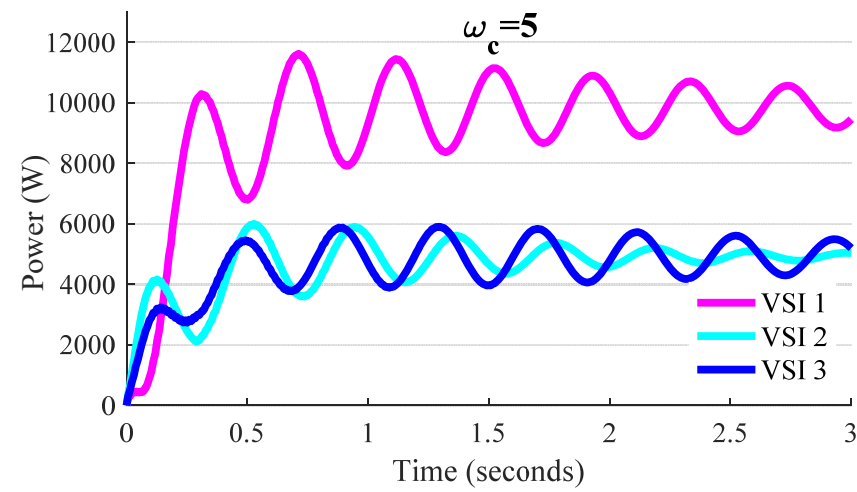

(a)

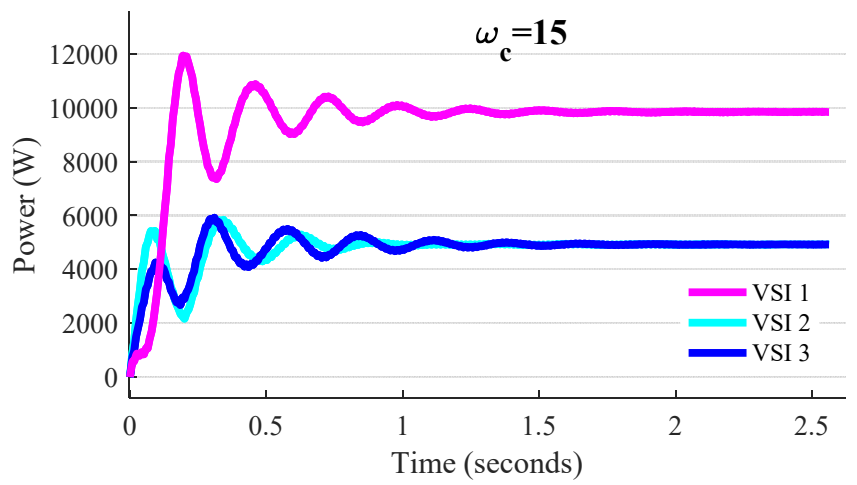

(b)

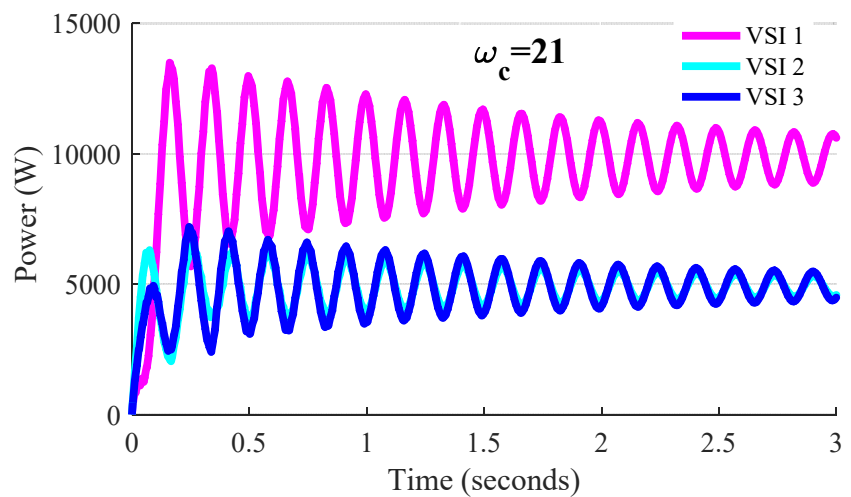

(c)

Fig. 13. Simulation results for different cut-off frequency $\omega_{c}$. (a) $\omega_{c}=5$. (b) $\omega_{\mathrm{c}}=15$. (c) $\omega_{\mathrm{c}}=21$.

\section{CONCLUSION}

In this paper, a comprehensive state-space model of the droop-based NMGs is proposed. The presented model is developed in a systematic way in order to enabling to obtain the small signal model of a partial or complete version of an NMG easily, without any concern about the topology of the power network. All important parts in the dynamic behavior of droopbased control system of an NMG are considered in the model which includes DC link, $f-P$ and $V-Q$ droop control loops, virtual impedance, VSI inner control loops, LC filter, local and common loads and power network. The main contribution of the presented model is that the power grid is modeled appropriately so that the interaction of droop control loops through the power network is properly evaluated. Simulation results are adopted to verify the accuracy of the presented model. In addition, the developed model is a reduced order model in comparison with conventional one, without losing the accuracy. Eigenvalue analyses of the corresponding state matrix of the developed model reveal some unstable regions, which are not discovered by conventional parallel-based model. The stability margins of the droop controlled-MG in relation to the variation of grid impedance $(X / R$ ratio to evaluate crosscoupling effect), increasing load demand (phase angle to evaluate the interaction of controllers) as well as cut-off frequency of the LPF (to evaluate the time constant of $f$ - $P$ loop) are discovered more accurately by the proposed model.

Although it is proven in this paper that power line dynamic is not an effective element on dynamic stability of droop-based NMGs, it is not a general agreement for low-voltage MGs with a fast-response control system which works based on high bandwidth communication network.

The proposed model can also be adopted for synthesis of advanced controllers provided that relevant communication link and measurements are available to secure controllability and observability of the model. Nevertheless, it remains as an open research topic to develop a less complex model for advanced control systems which is considered as the authors' future work noting that the VSI's inner loops dynamics cannot be neglected by simply ignoring relevant modes to PI controllers.

\section{Reference}

[1] R. H Lasseter, P. Paigi. "Microgrid: A conceptual solution." In Power Electronics Specialists Conference, 2004. PESC 04. 2004 IEEE 35th Annual. IEEE, 2004. 
[2] M. H. Moradi, M. Eskandari, S. M. Hosseinian, "Operational Strategy Optimization in an Optimal Sized Smart Microgrid", IEEE TSG, vol. 6, no. 3, pp. 1087-1095, 2015.

[3] N. Hatziargyriou, H. Asano, R. Iravani, C.Marnay. "Microgrids." IEEE power and energy magazine, vol. 5, no. 4, Pp. 78-94, Aug 2007.

[4] Tavakoli, Saman Dadjo, Mohammad Mahdavyfakhr, Mohsen Hamzeh, Keyhan Sheshyekani, and Ebrahim Afjei. "A unified control strategy for power sharing and voltage balancing in bipolar DC microgrids." Sustainable Energy, Grids and Networks 11 (2017): 58-68.

[5] M. H. Moradi, M. Eskandari, S. M. Hosseinian, "Cooperative control strategy of energy storage systems and micro sources for stabilizing microgrids in different operation modes," IJEPES, vol. 6, pp. 390-400, June. 2016.

[6] M. Eskandari and L. Li, "Microgrid operation improvement by adaptive virtual impedance," IET Renewable Power Generation, 2018.

[7] M. H. Moradi, M. Eskandari, and H. Showkati, "A hybrid method for simultaneous optimization of DG capacity and operational strategy in microgrids utilizing renewable energy resources," International Journal of Electrical Power \& Energy Systems, vol. 56, pp. 241-258, 2014.

[8] M. Eskandari, L. Li, and M. H. Moradi, "Improving power sharing in islanded networked microgrids using fuzzy-based consensus control," Sustainable Energy, Grids and Networks, 2018.

[9] Y. Pei, G. Jiang, and X. Yang, "Auto-master-slave control technique of parallel inverters in distributed AC power systems and UPS," in Proc. 35th Annu. IEEE Power Electron. Conf., Aachen, Germany, 2004, pp. 2050-2053.

[10] M. C. Chandorkar, D. M. Divan, and R. Adapa, "Control of parallel connected inverters in standalone AC supply systems," IEEE TIA., vol. 29, no. 1 , Jan 1993

[11] M. S. Sadabadi, Q. Shafiee, A. Karimi, "Plug-and-Play Voltage Stabilization in Inverter-Interfaced Microgrids via a Robust Control Strategy." IEEE Transactions on Control Systems Technology, vol 25, no. 3, pp. 781-791, May 2017.

[12] J. W. Simpson-Porco, F. Dörfler, and F. Bullo, "Voltage stabilization in microgrids via quadratic droop control," IEEE Transactions on Automatic Control, vol. 62, no. 3, pp. 1239-1253, 2017.

[13] J. M. Guerrero, J. C. Vasquez, and J. Matas, "Hierarchical control of droop controlled AC and DC microgrids-A general approach toward standardization,” IEEE TIE., vol. 58, no. 1, pp. 158-172, Jan. 2011.

[14] D. E. Olivares et al., "Trends in microgrid control," IEEE Trans. Smart Grid, vol. 5, no. 4, pp. 1905-1919, Jul. 2014.

[15] J. M. Guerrero, L. G. De Vicuna, J.Matas, M. Castilla, J. Miret. "A wireless controller to enhance dynamic performance of parallel inverters in distributed generation systems," IEEE TPE vol. 19, no. 5, pp. 1205-1213, Sep 2004.

[16] F. Katiraei and M. R. Iravani, "Power management strategies for a microgrid with multiple distributed generation units," IEEE Trans. Power Syst., vol. 21, no. 4, pp. 1821-1831, Nov. 2006.

[17] Y. A. I. Mohamed, E. F. El-Saadany. "Adaptive decentralized droop controller to preserve power sharing stability of paralleled inverters in distributed generation microgrids." IEEE TPE, vol. 23, no. 6, pp. 28062816, Nov. 2008

[18] Vasquez, Juan C., Josep M. Guerrero, Alvaro Luna, Pedro Rodríguez, and Remus Teodorescu. "Adaptive droop control applied to voltage-source inverters operating in grid-connected and islanded modes." IEEE TIE, vol. 56, no. 10, pp. Oct. 2009

[19] Chung, Il-Yop, Wenxin Liu, David A. Cartes, Emmanuel G. Collins, and Seung-Il Moon. "Control methods of inverter-interfaced distributed generators in a microgrid system." IEEE TIA, vol. 46, no. 3, pp.1078-1088, May 2010.

[20] R. Majumder, B. Chaudhuri, and A. Ghosh, "Improvement of stability and load sharing in an autonomous microgrid using supplementary droop control loop," IEEE Trans. Pwr Syst., vol. 25, no. 2, 796-808, May 2010.

[21] J. Kim, J. M. Guerrero, P. Rodriguez, R. Teodorescu, and K. Nam, "Mode adaptive droop control with virtual output impedances for an inverter-based flexible AC microgrid," IEEE Trans. Power Electron., vol. 26, no. 3, pp. 689-701, Mar. 2011

[22] J. He, Y. W. Li, "Analysis, Design, and Implementation of Virtual Impedance for Power Electronics Interfaced Distributed Generation," IEEE Trans. Industry Applications, vol. 47, no. 6, Nov 2011.
[23] Vasquez, Juan C., Josep M. Guerrero, Mehdi Savaghebi, Joaquin EloyGarcia, and Remus Teodorescu. "Modeling, analysis, and design of stationary-reference-frame droop-controlled parallel three-phase voltage source inverters." IEEE Transactions on Industrial Electronics, vol. 60, no. 4, pp. 1271-1280, Apr 2013

[24] J. Schiffer, T. Seel, J. Raisch, T. Sezi, "Voltage Stability and Reactive Power Sharing in Inverter-Based Microgrids with Consensus-Based Distributed Voltage Control," IEEE TCST, vol. 24, no. 10, pp. 96-109, January. 2016.

[25] Lin-Yu Lu, Chia-Chi Chu, "Consensus-Based Droop Control Synthesis for Multiple DICs in Isolated Micro-Grids," IEEE Trans. Power Syst., vol. 30, no. 5, pp. 2243-2256, Sep. 2015.

[26] H. Bevrani, M. R. Feizi, S. Ataee. "Robust Frequency Control in an

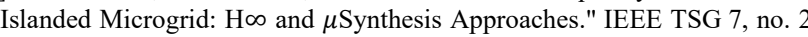
(2016): 706-717.

[27] M. Eskandari, L. Li, and M. H. Moradi, "Decentralized Optimal Servo Control System for Implementing Instantaneous Reactive Power Sharing in Microgrids," IEEE Transactions on Sustainable Energy, vol. 9, no. 2, pp. $525-537,2018$

[28] Baghaee, Hamid Reza, Mojtaba Mirsalim, Gevork B. Gharehpetian, and Heidar Ali Talebi. "A generalized descriptor-system robust $\mathrm{H} \infty$ control of autonomous microgrids to improve small and large signal stability considering communication delays and load nonlinearities." International Journal of Electrical Power \& Energy Systems 92 (2017): 63-82.

[29] E. A. A. Coelho, P. C. Cortizo, P. F. D. Garcia. "Small-signal stability for parallel-connected inverters in stand-alone AC supply systems." IEEE Trans. on Industry Applications, vol. 38, no. 2, pp. 533-542, Apr 2002

[30] E. A. A. Coelho, D. Wu, J. M. Guerrero, J. C. Vasquez, T. Dragicevi, C Stefanovi, P.Popovski, "Small-Signal Analysis of the Microgrid Secondary Control Considering a Communication Time Delay," IEEE TIE, vol. 63, no. 10, pp. 6257-6269, 2016

[31] Marwali, Mohammad N., Jin-Woo Jung, and Ali Keyhani. "Stability analysis of load sharing control for distributed generation systems." IEEE Transactions on Energy Conversion, vol. 22, no. 3, pp. 737-745, Sep 2007.

[32] S. V. Iyer, Madhu N. Belur, and Mukul C. Chandorkar. "A generalized computational method to determine stability of a multi-inverter microgrid." IEEE Transactions on Power Electronics, vol. 25, no. 9, pp. 2420-2432, Sep 2010

[33] F. Katiraei, M. R. Iravani, and P. W. Lehn. "Small-signal dynamic model of a micro-grid including conventional and electronically interfaced distributed resources." IET GTD, vol. 1, no. 3, pp. 369-378, May, 2007.

[34] W. Du, H. F. Wang, X. F. Zhang, and L. Y. Xiao. "Effect of gridconnected solid oxide fuel cell power generation on power systems smallsignal stability." IET RPG, vol. 6, no. 1, pp. 24-37, Jan 2012.

[35] X. Tang, W. Deng, Z. Qi. "Investigation of the dynamic stability of microgrid." IEEE TPWRS vol. 29, no. 2, pp. 698-706, Mar 2014.

[36] X. Guo, Zh. Lu, B. Wang, X. Sun, L. Wang,Josep M. Guerrero, "Dynamic Phasors-Based Modeling and StabilityAnalysis of DroopControlled Inverters for Microgrid Applications," IEEE Trans. Smart Grid, vol. 5, no. 6, pp. 2980-2987, Nov 2014.

[37] N. Bottrell, M. Prodanovic, T.C. Green. "Dynamic stability of microgrid with an active load." IEEE TPE, vol. 28, no. 11, pp. 5107-5119, Nov 2013.

[38] Shahnia, Farhad, and Ali Arefi. "Eigenanalysis-based small signal stability of the system of coupled sustainable microgrids." International Journal of Electrical Power \& Energy Systems 91 (2017): 42-60.

[39] N. Pogaku, M. Prodanovic', T. C. Green, "Modeling, Analysis and Testing of Autonomous Operation of an Inverter-Based Microgrid," IEEE Trans. Power Electron, vol. 22, no. 2, pp. 613-625, March 2007.

[40] Krismanto, Awan Uji, N. Mithulananthan, and Olav Krause. "Stability of Renewable Energy based Microgrid in Autonomous Operation." Sustainable Energy, Grids and Networks 13 (2018): 134-147.

[41] F Dheer, Dharmendra Kumar, Nimish Soni, and Suryanarayana Doolla. "Improvement of small signal stability margin and transient response in inverter-dominated microgrids." Sustainable Energy, Grids and Networks 5 (2016): 135-147.

[42] S. Leitner, M. Yazdanian, A. Mehrizi-Sani, A. Muetze. "Small-Signal Stability Analysis of an Inverter-Based Microgrid with Internal ModelBased Controllers." IEEE Transactions on Smart Grid (2017). 
[43] S. Tong and K. N. Miu, "A network-based distributed slack bus model for DGs in unbalanced power flow studies," IEEE Transactions on Power Systems, vol. 20, no. 2, pp. 835-842, 2005.

[44] M. Z. Kamh and R. Iravani, "A sequence frame-based distributed slack bus model for energy management of active distribution networks," IEEE Transactions on smart Grid, vol. 3, no. 2, pp. 828-836, 2012.

[45] A. Elrayyah, Y. Sozer, and M. E. Elbuluk, "A novel load-flow analysis for stable and optimized microgrid operation," IEEE Transactions on Power Delivery, vol. 29, no. 4, pp. 1709-1717, 2014.

[46] M. M. A. Abdelaziz, "Effect of detailed reactive power limit modeling on islanded microgrid power flow analysis," IEEE Transactions on Power Systems, vol. 31, no. 2, pp. 1665-1666, 2016.
[47] K. De Brabandere, "Voltage and frequency droop control in low voltage grids by distributed generators with inverter front- end," $\mathrm{Ph} . \mathrm{D}$. dissertation, Fac. Ingenieurswetenschappen, Katholieke Univ. Leuven, Leuven, Belgium, 2006.

[48] C. Kammer, "Frequency-domain controller design in power systems", $\mathrm{Ph}$. D. dissertation, Ecole polytechnique Federale de Lausanne, 2018.

[49] X. Guo, Z. Lu, B. Wang, X. Sun, L. Wang, and J. M. Guerrero, "Dynamic phasors-based modeling and stability analysis of droop-controlled inverters for microgrid applications," IEEE Transactions on Smart Grid, vol. 5, no. 6, pp. 2980-2987, 2014.

\section{Appendix:}

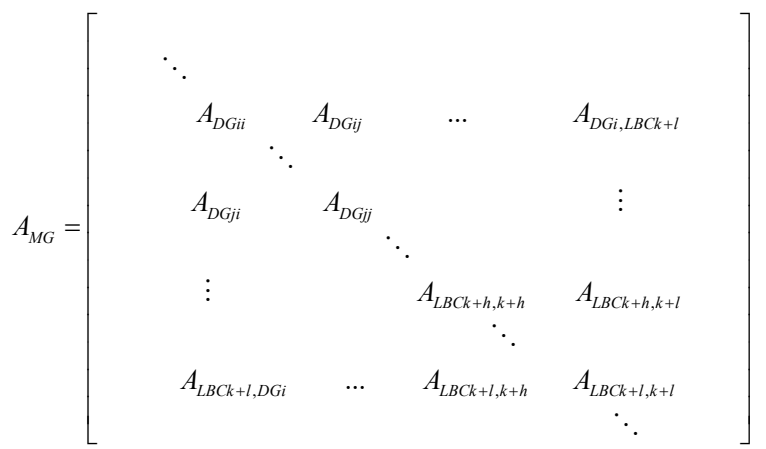

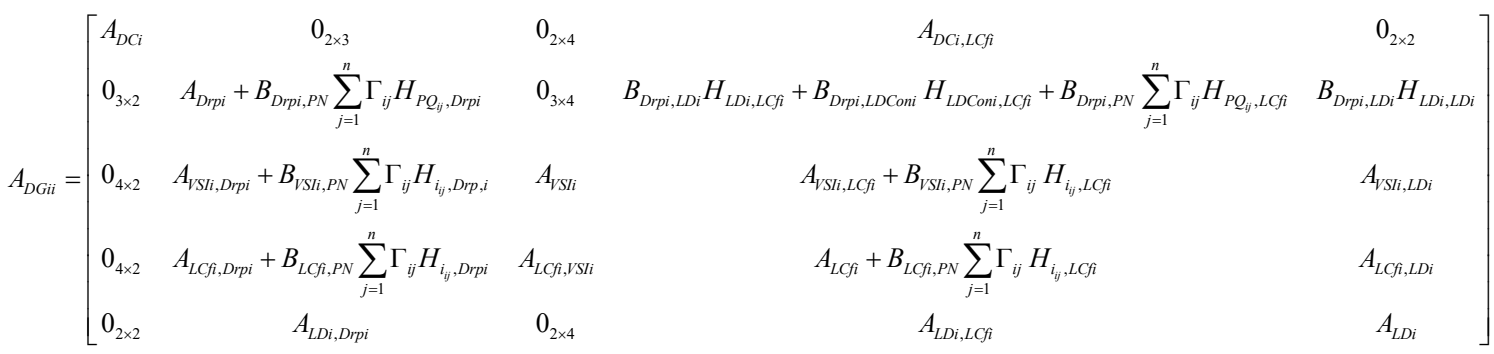

$$
\begin{aligned}
& A_{D G i j}=\Gamma_{i j}\left[\begin{array}{ccccc}
0_{2 \times 2} & 0_{2 \times 3} & 0_{2 \times 4} & 0_{2 \times 4} & 0_{2 \times 2} \\
0_{3 \times 2} & B_{D r p i, P N} H_{P Q_{i j}, D r p j} & 0_{3 \times 4} & B_{D r p i, P N} H_{P Q_{i j}, L C f j} & 0_{3 \times 2} \\
0_{4 \times 2} & B_{V S l i, P N} H_{i_{i j}, D r p j} & 0_{4 \times 4} & B_{V S S l i, P N} H_{i_{i j}, L C f j} & 0_{4 \times 2} \\
0_{4 \times 2} & B_{L C f i, P N} H_{i_{i j}, D r p j} & 0_{4 \times 4} & B_{L C f i, P N} H_{i_{i j}, L C f j} & 0_{4 \times 2} \\
0_{2 \times 2} & 0_{2 \times 3} & 0_{2 \times 4} & 0_{2 \times 4} & 0_{2 \times 2}
\end{array}\right], 1 \leq i, j \leq k \\
& A_{D G i, L B C j}=\Gamma_{i j}\left[\begin{array}{cc}
0_{2 \times 2} & 0_{2 \times 2} \\
B_{D r p i, P N} H_{P Q_{i j}, L B j} & 0_{3 \times 2} \\
B_{V S L I i, P N} H_{i_{i j}, L B j} & 0_{4 \times 2} \\
B_{L C f i, P N} H_{i_{i j}, L B j} & 0_{4 \times 2} \\
0_{2 \times 2} & 0_{2 \times 2}
\end{array}\right], 1 \leq i \leq k, k+1 \leq j \leq n \\
& A_{L B C i i}=\left[\begin{array}{cc}
A_{L B i}+B_{L B i, P N} \sum_{j=1}^{n} \Gamma_{i j} H_{i_{i j}, L B i} & A_{L B i, L D i} \\
A_{L D i, L B i} & A_{L D i}
\end{array}\right], k+1 \leq i \leq n, 1 \leq j \leq n \\
& A_{L B C i, D G j}=\Gamma_{i j}\left[\begin{array}{ccccc}
0_{2 \times 2} & B_{L B i, P N} H_{i_{i j}, D r p j} & 0_{2 \times 4} & B_{L B i, P N} H_{i_{i j}, L C f j} & 0_{2 \times 2} \\
0_{2 \times 2} & 0_{2 \times 3} & 0_{2 \times 4} & 0_{2 \times 4} & 0_{2 \times 2}
\end{array}\right], k+1 \leq i \leq n, 1 \leq j \leq k \\
& A_{L B C i j}=\Gamma_{i j}\left[\begin{array}{cc}
B_{L B i, P N} \sum_{j=1}^{n} \Gamma_{i j} H_{i_{i j}, L B j} & 0_{2 \times 2} \\
0_{2 \times 2} & 0_{2 \times 2}
\end{array}\right], k+1 \leq i, j \leq n
\end{aligned}
$$




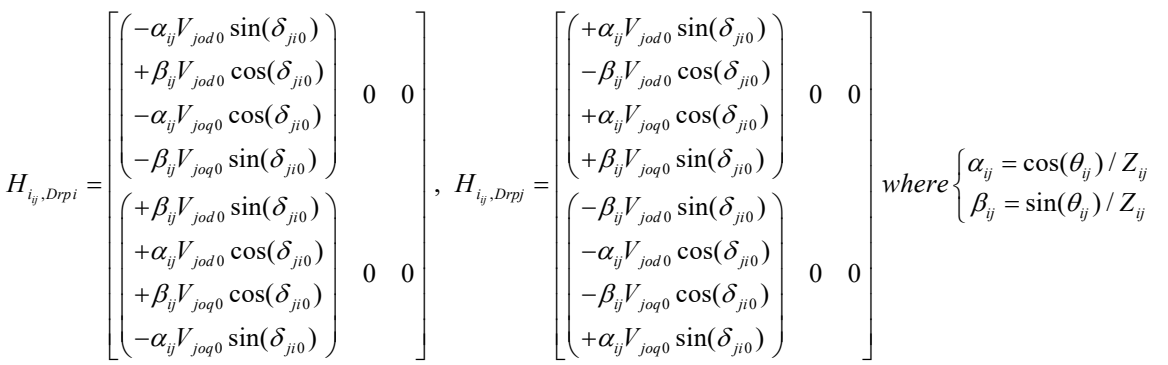

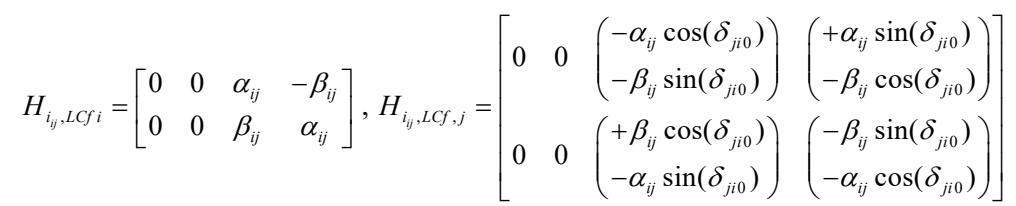

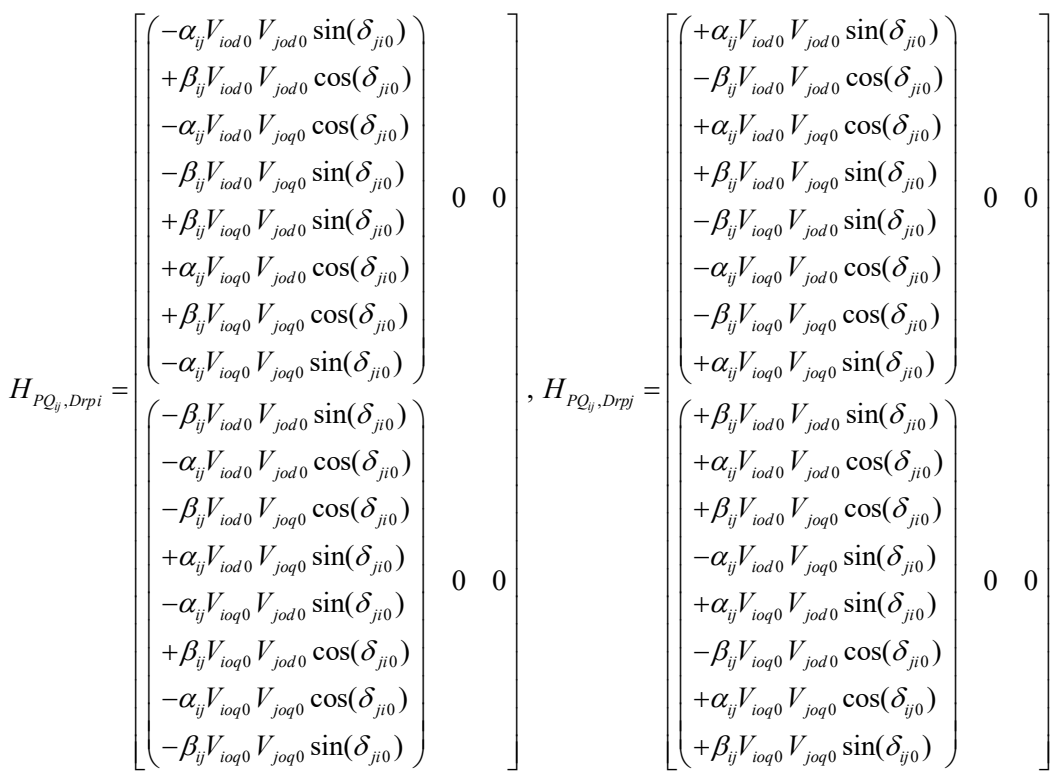

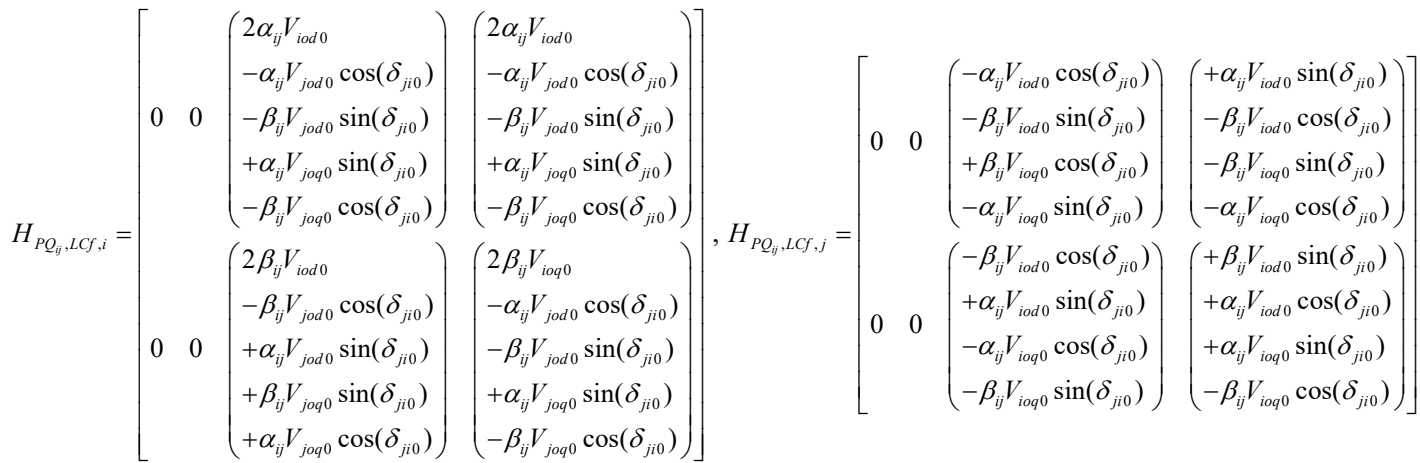



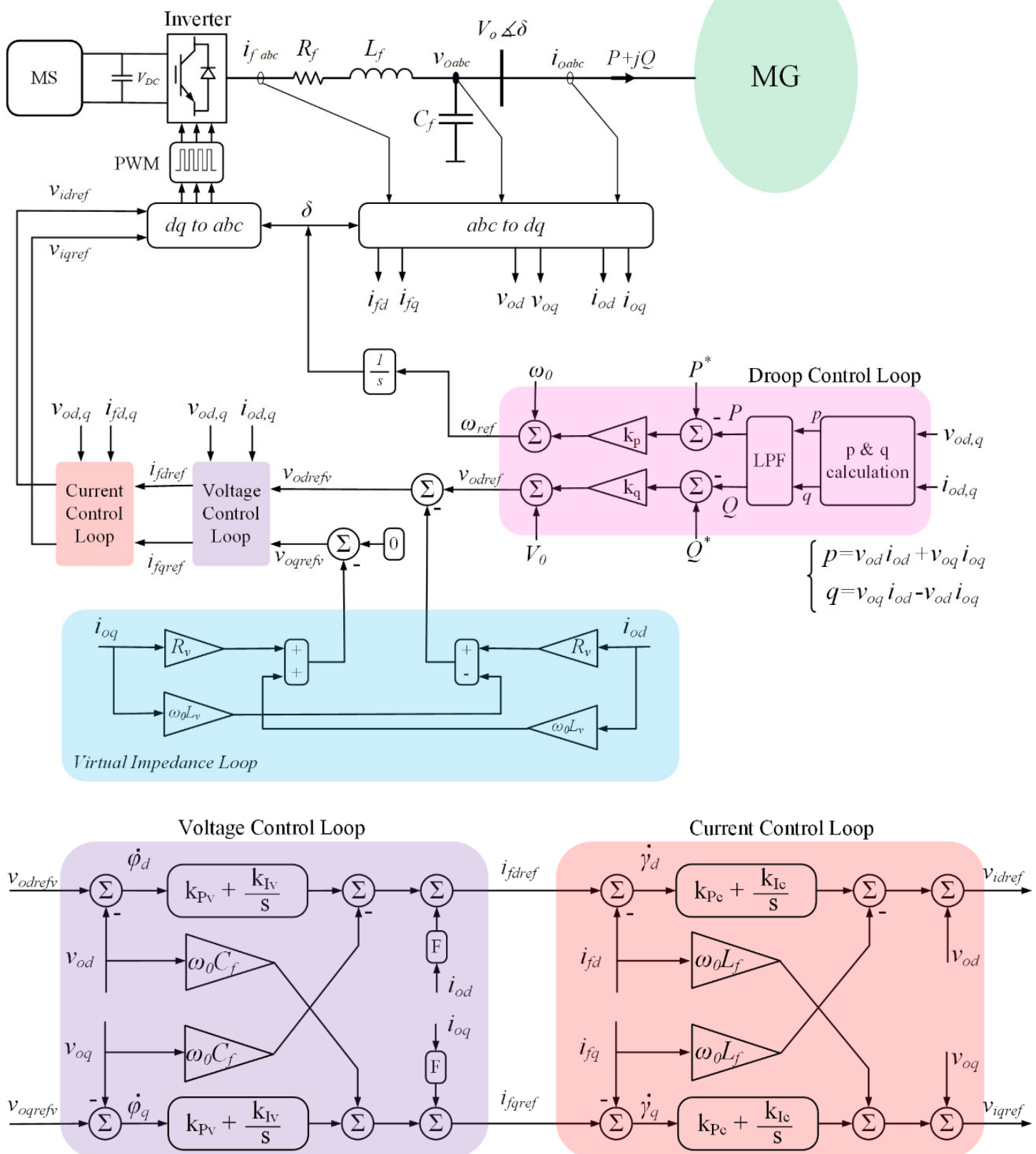

Fig. A1. VSI control system adopted for developing the Simulink model. 